\title{
Calculating the chiral condensate of QCD at infinite coupling using a generalised lattice diagrammatic approach
}

\author{
Alexander S. Christensen, ${ }^{a}$ Joyce C. Myers, ${ }^{a}$ Peter D. Pedersen ${ }^{a}$ and Jan Rosseel ${ }^{b}$ \\ ${ }^{a}$ Niels Bohr Institute, \\ Blegdamsvej 17, 2100 Copenhagen, Denmark \\ ${ }^{b}$ Vienna University of Technology, \\ Wiedner Hauptstr., 8-10/136, A-1040 Vienna, Austria \\ E-mail: xander@nbi.dk, jcmyers@nbi.dk, peter.pedersen@nbi.dk, \\ rosseelj@hep.itp.tuwien.ac.at
}

ABstract: We develop a lattice diagrammatic technique for calculating the chiral condensate of QCD at infinite coupling inspired by recent work of Tomboulis and earlier work from the 80 's. The technique involves calculating the contribution of gauge link diagrams formed from all possible combinations of a truncated number of sub-diagram types, by performing a resummation. We show how to calculate the relevant sub-diagrams, including a new technique for evaluating group integrals with arbitrary number of gauge link elements, using Young Projectors. Including up to four different diagram types we calculate the chiral condensate as a function of $N_{f}$, and show that two real solutions result, which are non-zero for all integer $N_{f}$. We analyse these solutions and find signs of convergence of the expansion at small $N_{f}$. We should stress that a drawback of our technique is that, due to the addition of non-tree diagrams in the resummation, there are sources of error associated with miscounting and over-counting of diagrams. We discuss these sources of error in detail, and implement a technique to reduce over-counting of diagrams, while leaving other sources of error for future work.

Keywords: Lattice QCD, Beyond Standard Model, Phase Diagram of QCD

ArXiv EPRINT: 1410.0541 


\section{Contents}

1 Introduction $\quad 2$

2 Expansion of $\langle\bar{\psi} \psi\rangle$ at $g=\infty$

$\begin{array}{lll}3 & \text { Building }\langle\bar{\psi} \psi\rangle \text { from irreducible diagrams } & 7\end{array}$

4 Fundamental base diagrams 11

$4.1 \quad L=1$ : ' $a$-type' 12

$4.2 L=4$ : 'b-type' 12

$\begin{array}{lll}4.3 L=6 & 12\end{array}$

4.3.1 'c-type' 12

4.3.2 $N_{c}=3:$ ' $d$-type' 13

$\begin{array}{lll}4.4 & L=7: \text { : } e \text {-type' } & 13\end{array}$

$\begin{array}{lll}4.5 & L=8 & 13\end{array}$

4.5.1 'f-type' 13

4.5.2 'g-type' 13

$\begin{array}{lll}4.6 & L=9 & 14\end{array}$

4.6.1 $N_{c}=3 \quad 14$

5 Calculating $\mathrm{SU}\left(N_{c}\right)$ group integrals $\quad 14$

$\begin{array}{ll}5.1 \text { General procedure } & 15\end{array}$

$\begin{array}{ll}5.2 & \text { Diagrammatic techniques }\end{array}$

6 Sources of error $\quad 23$

6.1 Mis-counting of overlapping graphs 23

$\begin{array}{lll}6.1 .1 & L=8 & 23\end{array}$

6.1.2 L $=12 \quad 24$

6.2 Avoiding over-counting of graphs 24

$\begin{array}{ll}\text { 6.2.1 Overlapping of } b \text {-type graphs } & 25\end{array}$

6.2.2 Avoiding over-counting of $b$-type graphs 26

$\begin{array}{lll}6.3 & \text { Over-counting resulting from symmetries } & 27\end{array}$

$\begin{array}{lll}\mathbf{7} & \text { Results } & \mathbf{2 8}\end{array}$

$\begin{array}{lll}7.1 & \text { Asymptotic solutions for large } N_{f} / N_{c} & 28\end{array}$

7.2 Numerical results for $N_{c}=3 \quad 31$

7.3 Comparison with lattice data 33

$\begin{array}{ll}7.4 & \text { Restricting to reduced graphs } \\ 75\end{array}$

8 Discussion and conclusions $\quad 37$

$\begin{array}{ll}\text { A Dimensionalities } & 39\end{array}$ 


\section{Introduction}

Until recently, it was thought that the chiral condensate of QCD at infinite coupling would remain non-zero for any number of fundamental fermion flavours $N_{f}$. This is in contrast to the restoration of chiral symmetry which is observed at some critical $N_{f}$ for more moderate couplings, resulting in the appearance of a conformal window (see for example [1-6] for a selection of lattice simulation results with fundamental representation fermions). The belief that the chiral symmetry remains broken for $g=\infty$ is based on the results of a few studies in the 80's. Among these is the work of [7], in which the authors calculate the normalized chiral condensate $\frac{1}{N_{f} N_{c}}\langle\bar{\psi} \psi\rangle$ from a $1 / d$ expansion. They obtain a non-zero result which is independent of $N_{f}$ for the first two orders in the expansion.

The approach in [7] is considered to be reliable. In the limit $N_{f} \rightarrow 0$ the normalized chiral condensate approaches the result in [8], which employed a quite different analytic lattice diagrammatic approach, up to $\mathcal{O}(1 / d)$ corrections. Subsequently, the diagrammatic lattice approach of [8] was extended in [9] by systematically removing certain diagrams which lead to over-counting. In this way the authors in [9] obtain a result for $\frac{1}{N_{f} N_{c}}\langle\bar{\psi} \psi\rangle$ as $N_{f} \rightarrow 0$, which is equivalent to that in [7], including the $\mathcal{O}(1 / d)$ corrections.

More recently, lattice simulations have been performed with $g=\infty$ and the chiral condensate was obtained as a function of $N_{f}$ [10]. Surprisingly these simulations on $4^{4}$ and $6^{4}$ lattices indicate that the chiral condensate drops discontinuously to a value close to zero at a critical value of $N_{f} \sim 13$ staggered flavours. These results are clearly in contrast with the results in [7] from the $1 / d$ expansion. Moreover, the authors of [10] also show that in contrast to their simulation results, a mean field calculation [11] of the critical temperature $T_{c}$, above which chiral symmetry is expected to be restored, gives a non-zero result for all $N_{f}$. The authors of [10] however argue that the discrepancy between the mean field and simulation results can be attributed to the fact that the $N_{f}$-dependence of contributions due to multiple meson hopping along a given a link and baryon loops is incorrectly taken into account in a mean field treatment. The simulations of [10] clearly beg the question whether the existing analytical calculations of the chiral condensate can be extended to take the $N_{f}$-dependence correctly into account and whether the transition observed in simulations can be reproduced analytically.

The first analytical calculation carried out for the purpose of obtaining the large $N_{f}$ dependence of the chiral condensate at infinite coupling appeared in [12], shortly after the simulation results of [10]. The analysis of [12] uses a lattice diagrammatic approach and suggests the presence of a possible transition in the chiral condensate at some critical $N_{f}$ at infinite coupling, as observed in the simulations of [10]. The approach used in [12] consists of performing a hopping expansion where the resummation includes a second type of "mesonic" graph (each bond in the diagram contains one gauge link $U$ and one gauge link $U^{\dagger}$ ), which contains a closed loop, contributing an $N_{f}$-dependence. The result of [12] 
is that the normalized chiral condensate is non-zero up to a critical value of $N_{f} \sim 10.7$ staggered flavours, beyond which only complex-valued solutions exist. This value is in reasonable agreement with the critical value of $N_{f} \sim 13$ obtained in [10]. While this result is encouraging, the solution for the chiral condensate of [12] is not entirely satisfactory. Indeed, the analysis of [12] leads to two solutions for the chiral condensate, one of which goes to the result in [9] as $N_{f} \rightarrow 0$. This solution however, increases as a function of $N_{f}$ before turning complex. ${ }^{1}$ This is in contrast to what is seen in [10] and other simulations, where the chiral condensate gradually decreases before sharply dropping to zero at the critical $N_{f}$.

This slight tension between the solution of [12] and the simulation results motivated the present work and led us to revisit the old work of $[8,9]$ to include $N_{f}$-dependent contributions. In this paper, we calculate $\frac{1}{N_{f} N_{c}}\langle\bar{\psi} \psi\rangle$ at infinite coupling by extending [8, 9] to include $N_{f}$-dependent contributions using a procedure inspired by [12]. Generalising [9], we enumerate different types of base diagrams and resum these in a hopping expansion, to form all possible diagrams made out of these building blocks, and from these obtain the chiral condensate. In order to take the $N_{f}$-dependence into account, we will, inspired by the technique of [12], also calculate diagrams built out of subdiagram types including internal loops, which come with factors of $N_{f}$. We will for instance extend [8,9] by including diagrams that can not only be built up from tree contributions, but also using an additional mesonic diagram, i.e. we consider diagrams which can be formed by combinations of

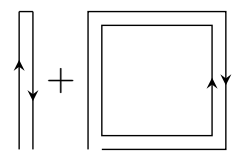

Note however that a calculation of the chiral condensate obtained by including only the diagrams formed from combinations of (1.1) will miss important contributions at sufficiently large $N_{f}$. For example, for $N_{c}=3$ we find that diagrams of the form

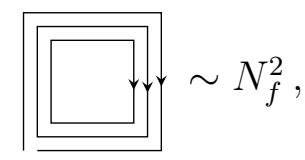

become important at larger $N_{f}$, even though diagrams formed out of base diagrams with more overlapping links appear to be suppressed in general (see the arguments at the end of section 3, as well as figure 5.). This suggests that it should be possible to improve results for the chiral condensate at larger $N_{f}$ by including diagrams with higher numbers of overlapping links which go like $N_{f}^{i}$ for $i>1$ and that adding up all possible nonzero link diagrams in a hopping expansion could lead to a precise value for the chiral condensate as a function of $N_{f}$. In this paper, we will make a selection of the most significant diagrams, up to a given order in the hopping expansion. Specifically, we focus on building diagrams out of area 1 (or less) sub-diagrams.

Our resummation of diagrams leads to a self-consistent system of equations for the chiral condensate. Up to the order at which we work, we find that there are multiple solutions

\footnotetext{
${ }^{1}$ The second solution decreases as a function of $N_{f}$, before turning complex at the same critical $N_{f}$ as the first solution, however, this one goes to infinity as $N_{f} \rightarrow 0$, contrary to simulation results.
} 
for the normalized chiral condensate as a function of $N_{f}$. Only one of these solutions has a sensible $N_{f} \rightarrow 0$ limit, matching onto the results of [7,9]. For small values of $N_{f}$, this solution behaves quantitatively reasonably well when compared with simulations and shows the expected gradual decrease. When considered for all values of $N_{f}$, we find that this solution for the chiral condensate approaches zero extremely slowly and that there is no sign of any sharp decline, discontinuity or of chiral symmetry restoration at any finite $N_{f}$, when restricting oneself to this solution alone. However, one can show that there is a second solution for the chiral condensate which is much larger at small $N_{f}$, and decreases more rapidly towards zero as $N_{f}$ increases. There is also no discontinuity or chiral symmetry restoration at any $N_{f}$ for the second solution. It cannot be ruled out that the chiral condensate jumps from one of these solutions to the other at some critical $N_{f}$. It is however not evident from our approach that a transition to chiral symmetry restoration occurs in this way.

We view the reasonable quantitative results at small $N_{f}$ as encouraging and as an indication that the work presented in this paper is a first step in the direction of obtaining a realistic analytical solution for the chiral condensate at infinite coupling. However, since we see no clear sign of a transition to chiral symmetry restoration (although such a transition cannot be excluded a priori), it is useful to comment on our approximations, namely the hopping expansion and the restriction to area 1 (or less) sub-diagrams. As we we will argue at the end of section 3, the validity of the hopping expansion, namely its convergence, follows if the normalised (by $1 / N_{c}$ ) chiral condensate does not become larger than 2 at any point. At $N_{f}=0$, there is indeed a solution for the chiral condensate which takes a value close to that determined in [9], around 0.66. This result is supported by the simulation results in [13], which agree at the level of a few percent. Since the chiral condensate is not expected to increase above its $N_{f}=0$ solution, the assumption that the normalized chiral condensate remains below 2 is natural, consistent with the decreasing magnitude of the chiral condensate observed in [13] and [10], as well as simulation results at more moderate coupling strength. This assumption is sufficient to argue that diagrams built out of the subdiagrams with less links contribute more, in principle, such that diagrams built out of lower area subdiagrams dominate. We note that there is always one solution which satisfies these assumptions. The reason for restricting ourselves to area 1 subdiagrams is further discussed at the end of section 3. There, it is argued mathematically that the area $n>1$ diagrams are generically of higher order in number of links, and that they are generically suppressed in comparison to the area 1 diagrams with the same $N_{f}$-dependence.

Let us stress that a drawback of lattice diagrammatic approaches of the type we use (and that are also used in [12]), where non-tree contributions are included, is that there are sources of error associated with over-counting, or with mis-counting of overlapping diagrams. This is discussed in section 6 , and to some degree we have been able to correct for these errors, however, not completely. In view of the simulation results, it would be interesting to investigate in detail whether removing these sources of error can lead to the presence of a transition at some critical $N_{f}$. We leave this for future work.

We can also compare our approach and results with those of [12]. Our resulting system of equations, which we have checked numerically including diagrams of up to 18 links, turns out to be different from that of [12]. The difference stems from the type of 
diagrams included and in how they are combined. To be specific, the analysis of [12] only includes mesonic diagrams (not including e.g. (1.2)) that are combinations of

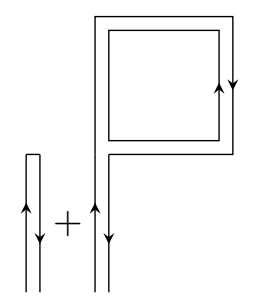

to be contrasted with (1.1). In our approach, we thus include additional diagrams formed by combining the building blocks of (1.1). We find that these have a significant effect on the chiral condensate. Specifically, when including all possible combinations of (1.3) within our approach, to form the same diagrams as in [12], we obtain qualitatively similar results, including a transition from real to complex solutions for the chiral condensate at some critical $N_{f}$. However, including diagrams which are left out in [12], by including all combinations of (1.1), causes this phenomenon to go away, leaving us with a chiral condensate which remains real, decreasing very slowly as a function of $N_{f}$, and only approaching zero as $N_{f} \rightarrow \infty$. This is discussed in more detail in section 7 .

As a technical by-product of this work, we will present a technique for evaluating group integrals, using Young projectors. Indeed, in order to calculate higher order diagrams with multiple overlapping gauge links $U$ and $U^{\dagger}$, it becomes necessary to evaluate $\mathrm{SU}\left(N_{c}\right)$ group integrals of the form

$$
\int_{\mathrm{SU}\left(N_{c}\right)} d U U_{a_{1}}^{b_{1}} \cdots U_{a_{m}}^{b_{m}}\left(U^{\dagger}\right)_{c_{1}}^{d_{1}} \cdots\left(U^{\dagger}\right)_{c_{n}}^{d_{n}}
$$

for some number of $U_{a}^{b},\left(U^{\dagger}\right)_{c}{ }^{d}$. We propose a simplified technique for evaluating this type of integral, using Young projectors. We comment on how this technique is related to previous approaches that appeared in [14-17].

The outline of this paper is as follows. In section 2, we will review how the chiral condensate at infinite coupling can be obtained from a lattice diagrammatic expansion [12]. In section 3, we will explain how the diagrammatic expansion can be resummed in a hopping expansion, that allows one to calculate the normalized chiral condensate from irreducible diagrams. Here, we generalize the analysis of [9], that only included $N_{f}$-independent tree graph contributions (that enclose zero area), to include irreducible diagrams that are built out of $N_{f}$-dependent base sub-diagrams that no longer lead to tree graphs. The relevant fundamental base sub-diagrams are given and calculated in section 4 . In section 5 we comment on various techniques to calculate $\mathrm{SU}\left(N_{c}\right)$ group integrals and explain a technique to evaluate these integrals in terms of Young projectors. In section 6, we discuss sources of error that are associated with our techniques and we show how over-counting of diagrams can be reduced. Our results are contained in section 7, where we also compare our methods with the ones used in [12] and with the simulation results in [13]. We conclude in section 8. 


\section{Expansion of $\langle\bar{\psi} \psi\rangle$ at $g=\infty$}

Our objective is to investigate the behaviour of the chiral condensate as a function of the number of fermion flavours $N_{f}$. To extend the procedure of obtaining $\frac{1}{N_{f} N_{c}}\langle\bar{\psi} \psi\rangle$ in [8, 9], for $N_{f} \rightarrow 0$, and in [12] for $N_{f} \neq 0$, to systematically account for the contributions which dominate in a diagrammatic expansion, order by order, it is necessary to understand how the diagrams contribute mathematically. Using the notation in [12], the chiral condensate $\langle\bar{\psi} \psi\rangle$ is obtained from

$$
\langle\bar{\psi}(x) \psi(x)\rangle=-\lim _{m \rightarrow 0} \partial_{m} \log Z,
$$

where the partition function $Z$ (after integrating out the fermion fields) is given by

$$
Z=\int d U \operatorname{det}\left[1+K^{-1} M(U)\right]
$$

with

$$
\begin{aligned}
M_{x y} & \equiv \frac{1}{2} \sum_{\mu}\left[\gamma_{\mu} U_{\mu}(x) \delta_{y, x+\hat{\mu}}-\gamma_{\mu} U_{\mu}^{\dagger}(x-\hat{\mu}) \delta_{y, x-\hat{\mu}}\right], \\
K_{x y} & =m \mathbb{I}_{N_{f}} \mathbb{I}_{N_{c}} \delta_{x y},
\end{aligned}
$$

for $\mu=1, \ldots, d$, including $N_{f}$ fermion flavours, and $N_{c}$ colours. The chiral condensate is thus given by [12]

$$
\langle\bar{\psi}(x) \psi(x)\rangle=-\lim _{m \rightarrow 0} \operatorname{tr}[G(x, x)]
$$

where

$$
G(x, x)=\frac{\int d U \operatorname{det}\left[1+K^{-1} M(U)\right]\left[\left[1+K^{-1} M(U)\right]^{-1} K^{-1}\right]_{x x}}{\int d U \operatorname{det}\left[1+K^{-1} M(U)\right]} .
$$

Expanding in powers of $K^{-1} M(U)$ one obtains

$$
\begin{aligned}
\operatorname{det}\left[1+K^{-1} M\right] & =\exp \operatorname{tr}\left[\sum_{n=1}^{\infty} \frac{(-1)^{n+1}}{n}\left(K^{-1} M\right)^{n}\right], \\
{\left[\left[1+K^{-1} M\right]^{-1} K^{-1}\right]_{x x} } & =\frac{1}{m}\left[\sum_{n=0}^{\infty}(-1)^{n}\left(K^{-1} M\right)^{n}\right]_{x x} .
\end{aligned}
$$

Note that $\operatorname{tr}$ [odd \# of $\gamma_{\mu}$ 's] $=0$ implies that only contributions from $\left(K^{-1} M\right)^{n}$ with $n$ even contribute to the integrals in (2.6). The trace in (2.7) (and (2.5)) extends over colour, flavour, and spinor degrees of freedom. For example,

$$
\begin{aligned}
& {\left[\left(K^{-1} M\right)^{2}\right]_{x x}=\frac{1}{(2 m)^{2}} \sum_{\mu, \nu} \sum_{y}\left[\gamma_{\mu} \gamma_{\nu}\right]} \\
& \quad \times\left[U_{\mu}(x) \delta_{y, x+\hat{\mu}}-U_{\mu}^{\dagger}(x-\hat{\mu}) \delta_{y, x-\hat{\mu}}\right]\left[U_{\nu}(y) \delta_{x, y+\hat{\nu}}-U_{\nu}^{\dagger}(y-\hat{\nu}) \delta_{x, y-\hat{\nu}}\right],
\end{aligned}
$$


and so on. In general, the trace in (2.7) leads to a closed loop of link variables, because the first and last lattice site are identified. Each loop also comes with a factor of $N_{f}$. The traces over the gamma matrices can be determined from

$$
\left\{\gamma_{\mu}, \gamma_{\nu}\right\}=2 \delta_{\mu \nu} \mathbb{I}_{N_{s}}
$$

where $\gamma_{\mu}$ are the Euclidean gamma matrices and $N_{s}$ denotes the number of spinor degrees of freedom.

It is also useful to notice that certain types of contributions will lead to cancellations with the denominator in (2.6). Since all diagrams resulting from the determinant are closed loops, the contributions to $\langle\bar{\psi} \psi\rangle$ which cancel are closed loop diagrams which can be disconnected from the path of gauge links beginning and ending at $x$. For example, in the diagram
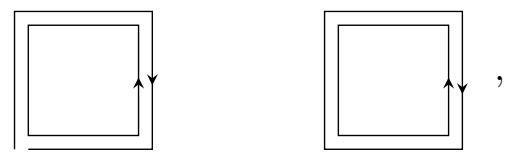

the closed loops on the right cancel with a contribution from the denominator. Note that this would even be true when there is partial overlap with links coming from $\left[\left[1+K^{-1} M\right]^{-1} K^{-1}\right]_{x x}$, as in

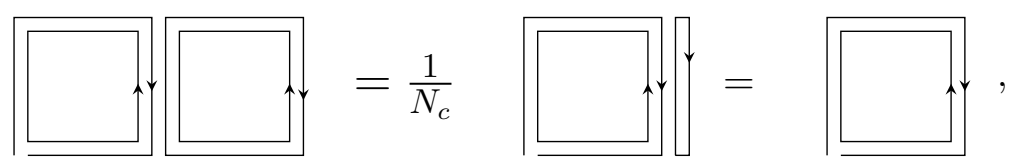

where the second equality is obtained by using

$$
U_{a}^{b}\left(U^{\dagger}\right)_{b}^{c}=\delta_{a}^{c}
$$

due to the unitarity of the U's. So one sees that, at least in some cases where there is partial overlap, the integrations can be separated.

\section{Building $\langle\bar{\psi} \psi\rangle$ from irreducible diagrams}

To generalise the diagram building procedure of [9] we calculate the chiral condensate (obtained from $\langle\bar{\psi} \psi\rangle=-\lim _{m \rightarrow 0} \operatorname{tr}[G(x, x)]$ ) by performing a hopping expansion, summing over gauge links order by order in the number of links

$$
\frac{\operatorname{tr}[G(x, x)]}{N_{s} N_{f} N_{c}}=\frac{1}{m} \sum_{L=0}^{\infty}(-1)^{L} \frac{A(L)}{(2 m)^{2 L}},
$$

where $A(L)$ is the contribution from all graphs with $2 L$ links which start and end at some site $x$. A general graph can be obtained by combining irreducible graphs $I(l)$ of $2 l$ links which start and end at $x$, where an irreducible graph is defined as one that cannot be separated into smaller segments which start and end at $x$. 


\section{Irreducible}

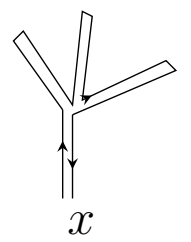

\section{Reducible}

The contribution $A(L)$ obeys the recursion relation

$$
A(L)=\sum_{l=1}^{L} I(l) A(L-l), \quad L \geq 1 ; \quad A(0)=1,
$$

where the irreducible graphs are built iteratively out of all possible combinations of smaller segments

$$
I(L)=2 d F_{0}(L-1)-4 d(d-1) \frac{N_{f}}{N_{c}} F_{1}(L-4)^{7}+\ldots,
$$

with $I(0)=0$, and the quantity $F_{n}(L)$ represents all possible graphs of length $2 L$ which start and end on a site on a sub-diagram of area $n$. It is given by

$$
F_{n}(L)=\sum_{\substack{l_{i}=1,2, \ldots, k_{j}=4,8, \ldots, \sum l_{i}+k_{j}=L-1}} I_{a}\left(l_{1}\right) I_{a}\left(l_{2}\right) \ldots I_{a}\left(l_{p}\right) I_{b}\left(k_{1}\right) I_{b}\left(k_{2}\right) \ldots I_{b}\left(k_{q}\right) \ldots \widehat{a}_{n}^{p} \widehat{b}_{n}^{q} \ldots
$$

with $F_{n}(0)=1$. In this formula, $I_{a}$ refers to irreducible graphs which begin with an ' $a$ type' sub-diagram, $\uparrow$, and $I_{b}$ refers to irreducible graphs which begin with a $L=4$ box, that is a 'b-type' sub-diagram, $\square$. Further types of sub-diagrams that can appear at larger $L$ will be denoted by 'c-type', ' $d$-type', $\ldots$ and will be defined later on in section 4 . In (3.4), we have also introduced the notation $\widehat{x}_{n} \equiv \frac{x_{n}}{d_{x}}$, where $x_{n}$ is the dimensionality of an attachment of type $x$ to an area $n$ diagram, and $d_{x}$ is the total dimensionality of a type $x$ diagram. These are catalogued in appendix A. For example,

$$
\begin{aligned}
& \widehat{a}_{0}=\frac{2 d-1}{2 d}, \\
& \widehat{b}_{0}=\frac{4(d-1)^{2}}{4 d(d-1)}=\frac{d-1}{d} .
\end{aligned}
$$

In particular, an $a$-type sub-diagram, $\uparrow$ attaches with dimensionality $2 d \widehat{a}_{n}$, to a graph of area $n$. All "tree" graphs are of this type (tree graphs don't include internal plaquettes). A $b$-type sub-diagram, $\Longrightarrow$ attaches with dimensionality $4 d(d-1) \widehat{b}_{n}$, to a graph of area $n$, such as $b$-type diagrams attached to $a$-type diagrams or other area 1 diagrams. The specific forms of $\widehat{a}_{n}, \widehat{b}_{n}, \ldots$ have been determined to avoid over-counting of graphs. ${ }^{2}$

\footnotetext{
${ }^{2}$ Regardless, there is some over-counting of attachments to certain winding diagrams, which will be discussed in section 6.3.
} 
As an illustration of (3.3) and (3.4), we note that the irreducible graphs $I(L)$ have the following form

$$
\begin{aligned}
& I(1)=\uparrow=I_{a}(1)=2 d, \\
& I(2)=\uparrow=I_{a}(2)=2 d\left[I_{a}(1) \widehat{a}_{0}\right],
\end{aligned}
$$
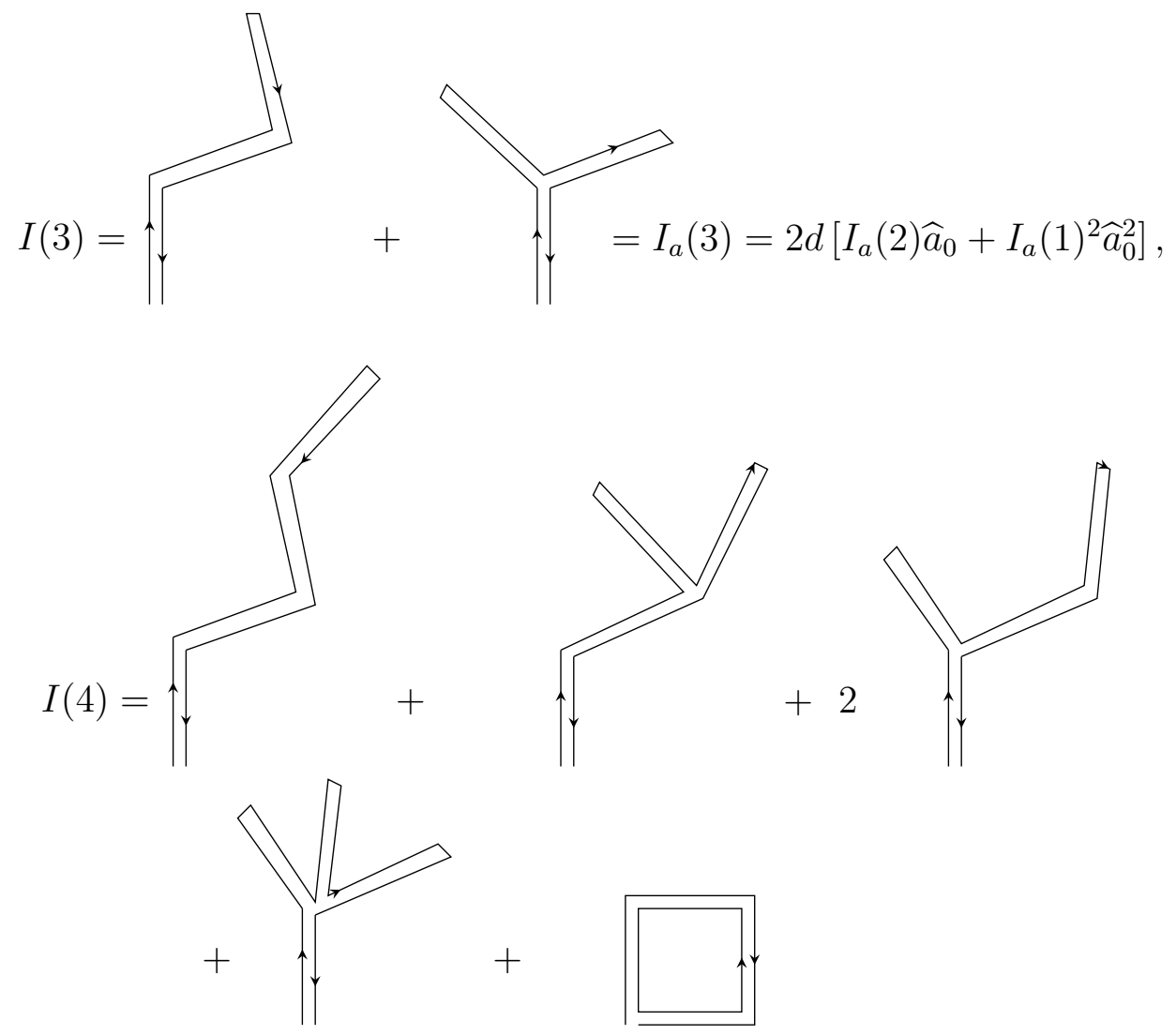

$$
=I_{a}(4)+I_{b}(4)
$$

$$
=2 d\left[I_{a}(3) \widehat{a}_{0}+2 I_{a}(1) I_{a}(2) \widehat{a}_{0}^{2}+I_{a}(1)^{3} \widehat{a}_{0}^{3}\right]-4 d(d-1) \frac{N_{f}}{N_{c}},
$$


The generating function, which gives the total contribution of all irreducible graphs including the mass dependence, is

$$
W_{I}=\sum_{l=0}^{\infty}\left(-\frac{1}{4 m^{2}}\right)^{l} I(l) .
$$

Using (3.3) for the $I(l)$ and defining $x=-\frac{1}{4 m^{2}}$ results in

$$
W_{I}=W_{a}+W_{b}+\ldots
$$

where $W_{a}$ is all irreducible graphs starting with an $a$-type base diagram $\uparrow, W_{b}$ is all irreducible graphs starting with a $b$-type base diagram

$$
\begin{aligned}
& W_{a}=2 d x \sum_{n=0}^{\infty}\left[\widehat{a}_{0} W_{a}+\widehat{b}_{0} W_{b}+\ldots\right]^{n}=\frac{2 d x}{1-\widehat{a}_{0} W_{a}-\widehat{b}_{0} W_{b}-\ldots}, \\
& W_{b}=-4 d(d-1) \frac{N_{f}}{N_{c}} x^{4}\left[\sum_{n=0}^{\infty}\left[\widehat{a}_{1} W_{a}+\widehat{b}_{1} W_{b}+\ldots\right]^{n}\right]^{7}=\frac{-4 d(d-1) \frac{N_{f}}{N_{c}} x^{4}}{\left(1-\widehat{a}_{1} W_{a}-\widehat{b}_{1} W_{b}-\ldots\right)^{7}},
\end{aligned}
$$

where the "..." include higher order (in $x$ ) base diagrams. The normalized chiral condensate is obtained by adding all possible combinations of irreducible graphs, such that

$$
\frac{\langle\bar{\psi} \psi\rangle}{N_{s} N_{f} N_{c}}=\lim _{m \rightarrow 0} \frac{\operatorname{tr}[G(x, x)]}{N_{s} N_{f} N_{c}}=\lim _{m \rightarrow 0} \frac{1}{m}\left(\frac{1}{1-W_{I}}\right) .
$$

In order to take the massless limit it is convenient to introduce the variables $g_{x} \equiv-\frac{2 m W_{x}}{d_{x}}$, for dimensional pre-factors $d_{a}=2 d, d_{b}=4 d(d-1), d_{c}=12 d(d-1)(2 d-3), \ldots$, such that the chiral condensate can be obtained from

$$
g \equiv d_{a} g_{a}+d_{b} g_{b}+\ldots,
$$

with, taking $m \rightarrow 0$,

$$
\begin{aligned}
g_{a} & =\frac{1}{a_{0} g_{a}+b_{0} g_{b}+\ldots}, \\
g_{b} & =\frac{\frac{N_{f}}{N_{c}}}{\left(a_{1} g_{a}+b_{1} g_{b}+\ldots\right)^{7}}, \\
g_{c} & =\frac{\frac{N_{f}}{N_{c}}}{\left(a_{2} g_{a}+b_{2} g_{b}+\ldots\right)^{11}}, \\
& \ldots,
\end{aligned}
$$

using

$$
\lim _{m \rightarrow 0} \frac{\operatorname{tr}[G(x, x)]}{N_{s} N_{f} N_{c}}=\frac{2}{g} .
$$


We derive the pre-factors $x_{n}$ in (3.19)-(3.22) in section 6.2. What we find is that the contributions to $g$ from the $g_{x}$ in general decrease in magnitude with increasing number of links in the base diagram (See figure 5 in section 7 ). Thus it appears that the series in (3.18) tends towards convergence.

A few comments are in order. First, it is useful to notice that for all $N_{f}$, diagram contributions with unit area will dominate over contributions with higher areas $n$. Since at leading order in $d$, the $x_{n}$ are the same for all $n$ and equivalent to $d_{x}$, then at this order the quantity $a_{n} g_{a}+b_{n} g_{b}+\ldots$ is independent of $n$ and equivalent to $d_{a} g_{a}+d_{b} g_{b}+\ldots$ In general the results in section 7 indicate that ${ }^{3}$

$$
g=d_{a} g_{a}+d_{b} g_{b}+\ldots>1
$$

This is already true at $N_{f} \rightarrow 0$, and the magnitude of $d_{a} g_{a}+d_{b} g_{b}+\ldots$ grows as a function of $N_{f}$, causing the magnitude of the chiral condensate to decrease. ${ }^{4}$ This implies that diagrams with a higher power of $\left(a_{n} g_{a}+b_{n} g_{b}+\ldots\right)^{-1}$ are suppressed at a fixed order in $N_{f}$. However, for sufficiently large $N_{f}$, diagrams which are higher order in $N_{f}$ will dominate regardless of whether they have higher powers of $\left(a_{n} g_{a}+b_{n} g_{b}+\ldots\right)^{-1}$. Therefore since larger areas result in more powers of $\left(a_{n} g_{a}+b_{n} g_{b}+\ldots\right)^{-1}$, at each order in $N_{f}$, the diagrams with the smallest area dominate.

In addition, the prefactors $x_{n}$ in the system of equations in (3.19)-(3.22) can be adjusted to reduce over-counting resulting from certain types of diagram attachments. The prefactors $x_{n}$ are derived in section 6.2, and tabulated in appendix A. These considerations are taken into account in the results for the normalized chiral condensate in section 7 .

\section{Fundamental base diagrams}

In this section we calculate the leading order fundamental base diagrams, from which irreducible graphs can be built. The contributions can be categorised based on the information in sections 2, 3. The calculations include the following components:

- A factor $\frac{1}{i !}\left(-N_{f} N_{s}\right)^{i}$, for a number $i$, of overlapping closed internal loops,

- A mass factor $\left(-\frac{1}{4 m^{2}}\right)^{n}$, for $n$ pairs of links,

- $(-1)^{k}$ for $k$ permutations of $\gamma$ matrices,

- $[\ldots]$, containing the result obtained by performing the group integrations,

- $\{\ldots\}$, containing the dimensionality of the graph.

\footnotetext{
${ }^{3}$ In general we find in section 7 that $g>1$ except at very small $N_{f}$ for solution 2 when working only to order $L=4$.

${ }^{4}$ This is consistent with lattice simulation results [10, 13], where the chiral condensate agrees well with [9] at $N_{f}=0$, then decreases in magnitude as a function of $N_{f}$.
} 
The group integrations can be performed using the techniques described in the next section (based on e.g. [14-17]). For this section, we will in particular need the expressions (5.7) and (5.14), that we repeat here for convenience:

$$
\begin{aligned}
& \int_{\mathrm{SU}\left(N_{c}\right)} d U U_{a}^{b}\left(U^{\dagger}\right)_{c}^{d}=\frac{1}{N_{c}} \delta_{a}^{d} \delta_{c}^{b} \\
& \int_{\mathrm{SU}\left(N_{c}\right)} d U U_{a_{1}}^{b_{1}} U_{a_{2}}^{b_{2}}\left(U^{\dagger}\right)_{c_{1}}^{d_{1}}\left(U^{\dagger}\right)_{c_{2}}^{d_{2}}=\frac{1}{2 N_{c}\left(N_{c}+1\right)}\left(\delta_{a_{1}}^{d_{1}} \delta_{a_{2}}^{d_{2}}+\delta_{a_{1}}^{d_{2}} \delta_{a_{2}}^{d_{1}}\right)\left(\delta_{c_{1}}^{b_{1}} \delta_{c_{2}}^{b_{2}}+\delta_{c_{1}}^{b_{2}} \delta_{c_{2}}^{b_{1}}\right) \\
& \quad+\frac{1}{2 N_{c}\left(N_{c}-1\right)}\left(\delta_{a_{1}}^{d_{1}} \delta_{a_{2}}^{d_{2}}-\delta_{a_{1}}^{d_{2}} \delta_{a_{2}}^{d_{1}}\right)\left(\delta_{c_{1}}^{b_{1}} \delta_{c_{2}}^{b_{2}}-\delta_{c_{1}}^{b_{2}} \delta_{c_{2}}^{b_{1}}\right)
\end{aligned}
$$

These integrals are sufficient to calculate diagrams with up to 4 overlapping links. In the next section, we will explain in more generality how group integrals can be calculated. The techniques explained there will enable us to also calculate diagrams that contain more than 4 overlapping links.

In the case of finite $N_{c}$, it is necessary to include additional 'baryonic' contributions, arising from integrals (5.25)

$$
\int_{\mathrm{SU}\left(N_{c}\right)} d U U_{a_{1}}^{b_{1}} \cdots U_{a_{N_{c}}}^{b_{N_{c}}}=\frac{1}{N_{c} !} \epsilon_{a_{1} \cdots a_{N_{c}}} \epsilon^{b_{1} \cdots b_{N_{c}}}
$$

In the following, we will list such contributions explicitly for the case $N_{c}=3$. We will moreover also restrict ourselves to the case of staggered fermions, for which $N_{s}=1$ and for which backtracking of the gauge links results in non-zero contributions.

The base diagrams up to order $L=9$ are as follows, where we also indicate the type the diagram belongs to.

\section{1 $L=1$ : ' $a$-type'}

$\eta=-\frac{1}{4 m^{2}}\{2 d\}$

\section{$4.2 L=4:$ ' $b$-type'}

$\square=\left(-\frac{1}{4 m^{2}}\right)^{4}(-1)^{2}\left(-N_{f}\right)\left[\frac{1}{N_{c}}\right]\{4 d(d-1)\}$

$4.3 L=6$

\subsection{1 'c-type'}

$=\left(-\frac{1}{4 m^{2}}\right)^{6}\left(-N_{f}\right)\left[\frac{1}{N_{c}}\right]\{12 d(d-1)(2 d-3)\}$ 
4.3.2 $N_{c}=3:$ ' $d$-type'

$$
\begin{aligned}
& \text { I }=\frac{1}{2 !}\left(-\frac{1}{4 m^{2}}\right)^{6}(-1)^{3}\left(-N_{f}\right)^{2}\left[\frac{1}{3}\right]\{4 d(d-1)\} \\
& =\left(-\frac{1}{4 m^{2}}\right)^{6}(-1)^{3}\left(-N_{f}\right)\left[-\frac{1}{3}\right]\{4 d(d-1)\} \\
& \square=\left(-\frac{1}{4 m^{2}}\right)^{6}(-1)^{3}\left[\frac{1}{3}\right]\{4 d(d-1)\} \\
& \square=\left(-\frac{1}{4 m^{2}}\right)^{6}(-1)^{3}\left(-N_{f}\right)\left[-\frac{1}{3}\right]\{4 d(d-1)\}
\end{aligned}
$$

$4.4 L=7$ : 'e-type'

$$
\left\{=\frac{1}{2 !}\left(-\frac{1}{4 m^{2}}\right)^{7}(-1)^{2}\left(-N_{f}\right)^{2}\left[\frac{1}{N_{c}^{2}}\right]\{12 d(d-1)(2 d-3)\}\right.
$$

$$
\longrightarrow
$$

$4.5 L=8$

\subsection{1 ' $f$-type'}

$$
\longrightarrow=\left(-\frac{1}{4 m^{2}}\right)^{8}(-1)^{2}\left(-N_{f}\right)\left[\frac{1}{N_{c}}\right]\left\{48 d(d-1)(2 d-3)^{2}\right\}
$$

\subsection{2 ' $g$-type'}

$$
\begin{aligned}
& \text { W }=\frac{3}{3 !}\left(-\frac{1}{4 m^{2}}\right)^{8}(-1)^{4}\left(-N_{f}\right)^{3}\left[\frac{2}{N_{c}}\right]\{4 d(d-1)\} \\
& \square=\frac{2}{2 !}\left(-\frac{1}{4 m^{2}}\right)^{8}(-1)^{4}\left(-N_{f}\right)^{2}[0]\{4 d(d-1)\} \\
& \square=\left(-\frac{1}{4 m^{2}}\right)^{8}(-1)^{4}\left(-N_{f}\right)\left[\frac{2}{N_{c}}\right]\{4 d(d-1)\}
\end{aligned}
$$




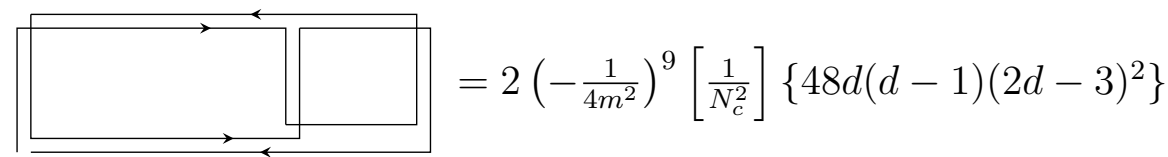

4.6.1 $\quad N_{c}=3$
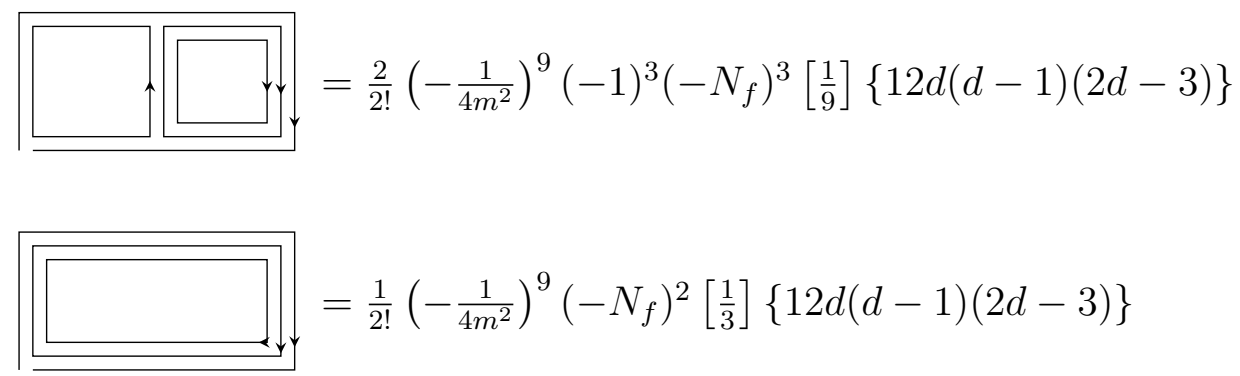

We note that in this enumeration there are base diagrams which contribute at $N_{f}=0$, in (4.9), (4.12), and (4.18), which are not decomposable into smaller diagrams, and which were not included in previous studies $[8,9,12]$. We note that at all orders diagrams of this type can be constructed, which cannot be decomposed into smaller diagrams. For instance, one can simply stretch out any of the diagrams above to create new higher order base diagrams which are not decomposable. These contributions are all suppressed compared to diagrams composed of tree sub-diagrams alone, that is, diagrams formed only by combinations of (4.4). Diagrams composed of sub-diagrams of the form (4.9) are suppressed by $1 / N_{c}$ per base diagram, compared to those formed with combinations of (4.4). Diagrams composed of sub-diagrams of the form (4.12), (4.18) are suppressed by $1 / N_{c}^{2}$ per base diagram, compared to those formed with combinations of (4.4). In this study we include contributions from sub-diagrams of the form (4.9) in our calculations, but not those of the form (4.12), (4.18), since these are area 2 and 3 diagrams. Our results indicate that including sub-diagrams of the type (4.9) has a barely observable effect. The normalised chiral condensate as $N_{f} \rightarrow 0$, goes from 0.66144 including tree diagrams alone [9], to 0.66139 including the contributions from sub-diagrams of the form (4.9) as well.

\section{Calculating $\mathrm{SU}\left(N_{c}\right)$ group integrals}

To obtain diagrams up to $\mathcal{O}\left(\left(\frac{1}{m^{2}}\right)^{16}\right)$, we need the following additional group integrals for general number of colours $N_{c}$

$$
\int_{\mathrm{SU}\left(N_{c}\right)} d U U_{a_{1}}^{b_{1}} U_{a_{2}}^{b_{2}} U_{a_{3}}^{b_{3}}\left(U^{\dagger}\right)_{c_{1}}^{d_{1}}\left(U^{\dagger}\right)_{c_{2}}^{d_{2}}\left(U^{\dagger}\right)_{c_{3}}^{d_{3}}
$$




$$
\int_{\mathrm{SU}\left(N_{c}\right)} d U U_{a_{1}}^{b_{1}} U_{a_{2}}^{b_{2}} U_{a_{3}}^{b_{3}} U_{a_{4}}^{b_{4}}\left(U^{\dagger}\right)_{c_{1}}^{d_{1}}\left(U^{\dagger}\right)_{c_{2}}^{d_{2}}\left(U^{\dagger}\right)_{c_{3}}^{d_{3}}\left(U^{\dagger}\right)_{c_{4}}^{d_{4}}
$$

Moreover, since we are interested in the case $N_{c}=3$, the following integrals also give a non-zero contribution at this order

$$
\begin{aligned}
& \int_{\mathrm{SU}(3)} d U U_{a_{1}}^{b_{1}} U_{a_{2}}^{b_{2}} U_{a_{3}}^{b_{3}} U_{a_{4}}^{b_{4}}\left(U^{\dagger}\right)_{c_{1}}^{d_{1}}, \quad \int_{\mathrm{SU}(3)} d U U_{a_{1}}^{b_{1}} U_{a_{2}}^{b_{2}} U_{a_{3}}^{b_{3}} U_{a_{4}}^{b_{4}} U_{a_{5}}^{b_{5}} U_{a_{6}}^{b_{6}} \\
& \int_{\mathrm{SU}(3)} d U U_{a_{1}}^{b_{1}} U_{a_{2}}^{b_{2}} U_{a_{3}}^{b_{3}} U_{a_{4}}^{b_{4}} U_{a_{5}}^{b_{5}}\left(U^{\dagger}\right)_{c_{1}}^{d_{1}}\left(U^{\dagger}\right)_{c_{2}}^{d_{2}}
\end{aligned}
$$

In this section, we will explain how integrals of this type can be calculated in full generality. Methods to calculate integrals of this type have appeared in the literature at various occasions (see e.g. [14-17]). In this section, we will employ a method that is loosely based on techniques that appeared in [16] and that, to our knowledge, has not yet appeared in the literature. It uses tensor product decompositions to write the required integrals in terms of Young projectors. It has the advantage that it can easily be implemented using a symbolic computer algebra system. This method, that we will explain in section 5.1 can be used to perform the group integrations associated to general diagrams. Diagrammatic methods to do these group integrations are given in [15]. For more complicated diagrams, these can quickly become cumbersome. For relatively simple diagrams, they can however be quick and useful, so we will give a brief summary of these techniques in section 5.2.

\subsection{General procedure}

In order to calculate the diagrams considered in this work, we need to evaluate various integrals of products of matrix elements of $\mathrm{SU}\left(N_{c}\right)$ group elements. Let us first focus on integrals of the form

$$
I_{m}=\int_{\mathrm{SU}\left(N_{c}\right)} d U U_{a_{1}}^{b_{1}} \cdots U_{a_{m}}^{b_{m}}\left(U^{\dagger}\right)_{c_{1}}^{d_{1}} \cdots\left(U^{\dagger}\right)_{c_{m}}^{d_{m}}
$$

where $U$ represents a $\mathrm{SU}\left(N_{c}\right)$ group element in the fundamental representation. Integrals of this form were calculated in an implicit manner in [14], where an iterative way of calculating the quantities

$$
F_{m}(A)=\int_{\mathrm{SU}\left(N_{c}\right)} d U(\operatorname{tr} A U)^{m}\left(\operatorname{tr} A^{\dagger} U^{\dagger}\right)^{m}
$$

for an arbitrary, constant matrix $A$, was given. In particular, it was argued that $F_{m}(A)$ is a linear combination of $\left(\operatorname{tr}\left(A A^{\dagger}\right)\right)^{k} \operatorname{tr}\left(A A^{\dagger}\right)^{m-k}$ (for $\left.k=0, \cdots, m\right)$ and that the coefficients of the linear combination can be obtained from knowledge of $F_{1}(A), \cdots, F_{m-1}(A)$. Once such an expression for $F_{m}(A)$ is obtained, it can be used to extract the integral (5.3), by writing out all traces explicitly in terms of matrix elements and Kronecker delta symbols. The integral (5.3) can then be found in terms of Kronecker delta symbols as the coefficient of $A_{b_{1}}^{a_{1}} \cdots A_{b_{m}}^{a_{m}}\left(A^{\dagger}\right)_{d_{1}}^{c_{1}} \cdots\left(A^{\dagger}\right)_{d_{m}}^{c_{m}}$, as can be seen by writing

$$
\int_{\mathrm{SU}\left(N_{c}\right)} d U(\operatorname{tr} A U)^{m}\left(\operatorname{tr} A^{\dagger} U^{\dagger}\right)^{m}=
$$




$$
\sum_{a_{i}, b_{i}, c_{i}, d_{i}} A_{b_{1}}^{a_{1}} \cdots A_{b_{m}}^{a_{m}}\left(A^{\dagger}\right)_{d_{1}}^{c_{1}} \cdots\left(A^{\dagger}\right)_{d_{m}}^{c_{m}} \int_{\mathrm{SU}\left(N_{c}\right)} d U U_{a_{1}}^{b_{1}} \cdots U_{a_{m}}^{b_{m}}\left(U^{\dagger}\right)_{c_{1}}^{d_{1}} \cdots\left(U^{\dagger}\right)_{c_{m}}^{d_{m}}
$$

Note that in extracting the integral (5.3) in this way, care has to be taken of making sure that the result has the correct symmetry properties for the indices. In particular, various symmetrizations have to be performed by hand. While in principle this gives a straightforward way to calculate the integrals (5.3), calculating the $F_{m}(A)$ and extracting the wanted integrals from it can be cumbersome, especially as $m$ gets larger. For the purpose of this paper, we will therefore use a different method, that allows one to directly and explicitly construct the integrals $I_{m}$, in a way that can be easily implemented using a symbolic computer program. We have explicitly checked that the results we get for $I_{m}$ agree with the results one can get from the formulas of [14] for $m=1, \cdots, 4$. We will now outline our method and illustrate it in two examples.

The general procedure to evaluate $I_{m}$ consists of the following steps:

1. First, one writes the decomposition of $m$ fundamental representations. This decomposition is given by the sum of all standard Young tableaux with $m$ entries.

2. Next, one constructs the Young projectors associated with the standard Young tableaux that appear in this decomposition. These Young projectors can be constructed by symmetrizing the expression $\delta_{a_{1}}^{b_{1}} \cdots \delta_{a_{m}}^{b_{m}}$ in the $a_{i}$-indices of the first row of the Young tableau. The resulting expression is then symmetrized in the $a_{i}$-indices appearing in the second row of the Young tableau and one continues this symmetrization procedure for all rows (from top to bottom). The result of this symmetrization is then antisymmetrized in the $a_{i}$-indices that appear in the first column of the tableau and similarly for all columns (from left to right). The Young projector is given by the result of these consecutive symmetrizations and antisymmetrizations, multiplied by a factor that is the inverse of the product of all hook lengths of the tableau. This factor guarantees that the Young projector squares to itself.

3. Using the decomposition of step 1, the integral (5.3) can be turned into a sum of integrals that are schematically of the form [16]

$$
\int_{\mathrm{SU}\left(N_{c}\right)} d U R_{\alpha}^{\beta}\left(S^{\dagger}\right)_{\gamma}^{\delta}=\frac{1}{d_{R}} \mathbb{P}_{\alpha}^{R \bar{\delta}} \mathbb{P}_{\gamma}^{S \bar{\beta}} \delta_{R, S}
$$

In this formula $R$ and $S$ are irreducible representations, that correspond to standard Young tableaux in the tensor product of $m$ fundamental representations. The dimension of $R$ has been denoted by $d_{R}$, while $\mathbb{P}_{\alpha}^{R \beta}$ corresponds to the Young projector that picks out the representation $R$ in the tensor product. The $\delta_{R, S}$ indicates that the above integral is only non-zero when $R, S$ correspond to representations with the same Young tableau shape. Note that we have used a schematic notation for the indices $\alpha, \beta, \gamma, \delta$ of the matrix elements of $R$ and $S$. These indices are composite and consist of $m$ indices in the fundamental representation, with symmetry properties indicated by the standard Young tableau that corresponds to $R$ or $S$. Note 
that in (5.6), the composite index $\delta$ has symmetry properties indicated by the Young tableau corresponding to $S$, whereas it has to appear in the Young projector corresponding to $R$. In case $R$ and $S$ correspond to different standard Young tableaux, one must reorder the indices that make up the composite index $\delta$ in such a way that the reordered collection, indicated by $\bar{\delta}$ in (5.6), has symmetry properties of the Young tableau that corresponds to $R$. Such a reordering is possible for Young tableaux with the same shape. An analogous remark holds for the composite index $\beta$.

All integrals $I_{m}$ can be calculated along the lines described above. The simplest integral is of course $I_{1}$, which by directly applying (5.6) is given by

$$
I_{1}=\int_{\mathrm{SU}\left(\mathrm{N}_{c}\right)} d U U_{a}^{b}\left(U^{\dagger}\right)_{c}^{d}=\frac{1}{N_{c}} \delta_{a}^{d} \delta_{c}^{b} .
$$

Let us now illustrate the above procedure via the calculation of $I_{2}$ and $I_{3}$.

Consider first the integral $I_{2}$

$$
I_{2}=\int_{\mathrm{SU}\left(N_{c}\right)} d U U_{a_{1}}^{b_{1}} U_{a_{2}}^{b_{2}}\left(U^{\dagger}\right)_{c_{1}}^{d_{1}}\left(U^{\dagger}\right)_{c_{2}}^{d_{2}}
$$

Since $U_{a_{1}}^{b_{1}} U_{a_{2}}^{b_{2}}$ acts in the tensor product of two fundamental representations $\left(a_{1} \otimes a_{2}\right)$ and since

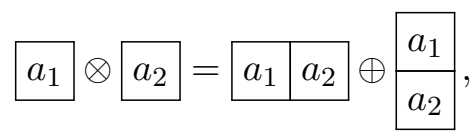

we can write

$$
U_{a_{1}}^{b_{1}} U_{a_{2}}^{b_{2}}=S_{a_{1} a_{2}}^{b_{1} b_{2}}+A_{a_{1} a_{2}}^{b_{1} b_{2}}
$$

where $S_{a_{1} a_{2}}^{b_{1} b_{2}}$ acts in the representation \begin{tabular}{|l|l|l|l|}
$a_{1}$ & $a_{2}$
\end{tabular} and $A_{a_{1} a_{2}}^{b_{1} b_{2}}$ acts in the representation \begin{tabular}{|l|l}
$a_{1}$ \\
\hline$a_{2}$
\end{tabular} . The symmetric and antisymmetric representation matrices $S_{a_{1} a_{2}}^{b_{1} b_{2}}, A_{a_{1} a_{2}}^{b_{1} b_{2}}$ can be obtained explicitly via

$$
\begin{aligned}
& S_{a_{1} a_{2}}^{b_{1} b_{2}}=\mathbb{P}_{a_{1} a_{2}}^{S c_{1} c_{2}}\left(U_{c_{1}}^{d_{1}} U_{c_{2}}^{d_{2}}\right) \mathbb{P}_{d_{1} d_{2}}^{S b_{1} b_{2}}, \\
& A_{a_{1} a_{2}}^{b_{1} b_{2}}=\mathbb{P}_{a_{1} a_{2}}^{A c_{1} c_{2}}\left(U_{c_{1}}^{d_{1}} U_{c_{2}}^{d_{2}}\right) \mathbb{P}_{d_{1} d_{2}}^{A b_{1} b_{2}},
\end{aligned}
$$

where the Young projectors $\mathbb{P}_{a_{1} a_{2}}^{S} b_{1} b_{2}, \mathbb{P}_{a_{1} a_{2}}^{A} b_{1} b_{2}$ on the symmetric and anti-symmetric representations are given by

$$
\begin{aligned}
& \mathbb{P}_{a_{1} a_{2}}^{S b_{1} b_{2}}=\frac{1}{2}\left(\delta_{a_{1}}^{b_{1}} \delta_{a_{2}}^{b_{2}}+\delta_{a_{1}}^{b_{2}} \delta_{a_{2}}^{b_{1}}\right) \\
& \mathbb{P}_{a_{1} a_{2}}^{A b_{1} b_{2}}=\frac{1}{2}\left(\delta_{a_{1}}^{b_{1}} \delta_{a_{2}}^{b_{2}}-\delta_{a_{1}}^{b_{2}} \delta_{a_{2}}^{b_{1}}\right)
\end{aligned}
$$

Using the decomposition (5.10), the integral (5.8) can be written as a sum of four terms

$$
I_{2}=\int_{\mathrm{SU}\left(N_{c}\right)} d U S_{a_{1} a_{2}}^{b_{1} b_{2}}\left(S^{\dagger}\right)_{c_{1} c_{2}}^{d_{1} d_{2}}+\int_{\mathrm{SU}\left(N_{c}\right)} d U A_{a_{1} a_{2}}^{b_{1} b_{2}}\left(A^{\dagger}\right)_{c_{1} c_{2}}^{d_{1} d_{2}}
$$




$$
+\int_{\mathrm{SU}\left(N_{c}\right)} d U S_{a_{1} a_{2}}^{b_{1} b_{2}}\left(A^{\dagger}\right)_{c_{1} c_{2}}^{d_{1} d_{2}}+\int_{\mathrm{SU}\left(N_{c}\right)} d U A_{a_{1} a_{2}}^{b_{1} b_{2}}\left(S^{\dagger}\right)_{c_{1} c_{2}}^{d_{1} d_{2}}
$$

The last two terms involve an integral of a product of two representations with different Young tableau shape and are therefore zero according to (5.6). The first two terms can be evaluated using the same rule, resulting in

$$
I_{2}=\frac{2}{N_{c}\left(N_{c}+1\right)} \mathbb{P}_{a_{1} a_{2}}^{S \quad d_{1} d_{2}} \mathbb{P}_{c_{1} c_{2}}^{S b_{1} b_{2}}+\frac{2}{N_{c}\left(N_{c}-1\right)} \mathbb{P}_{a_{1} a_{2}}^{A d_{1} d_{2}} \mathbb{P}_{c_{1} c_{2}}^{A b_{1} b_{2}}
$$

As a slightly more involved example, let us also consider the integral

$$
I_{3}=\int_{\mathrm{SU}\left(N_{c}\right)} d U U_{a_{1}}^{b_{1}} U_{a_{2}}^{b_{2}} U_{a_{3}}^{b_{3}}\left(U^{\dagger}\right)_{c_{1}}^{d_{1}}\left(U^{\dagger}\right)_{c_{2}}^{d_{2}}\left(U^{\dagger}\right)_{c_{3}}^{d_{3}}
$$

In this case, we can use the decomposition

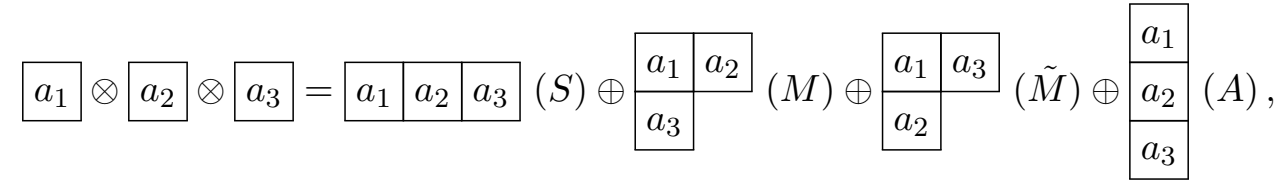

where in brackets we have given a shorthand notation to denote the corresponding tableaux, to write

$$
U_{a_{1}}^{b_{1}} U_{a_{2}}^{b_{2}} U_{a_{3}}^{b_{3}}=S_{a_{1} a_{2} a_{3}}^{b_{1} b_{2} b_{3}}+M_{a_{1} a_{2} a_{3}}^{b_{1} b_{2} b_{3}}+\tilde{M}_{a_{1} a_{2} a_{3}}^{b_{1} b_{2} b_{3}}+A_{a_{1} a_{2} a_{3}}^{b_{1} b_{2} b_{3}},
$$

where $S_{a_{1} a_{2} a_{3}}^{b_{1} b_{2} b_{3}}, M_{a_{1} a_{2} a_{3}}^{b_{1} b_{2} b_{3}}, \tilde{M}_{a_{1} a_{2} a_{3}}^{b_{1} b_{2} b_{3}}, A_{a_{1} a_{2} a_{3}}^{b_{1} b_{2} b_{3}}$ act in the representations indicated by the Young tableaux on the right-hand-side of eq. (5.16). They are explicitly obtained by acting with the appropriate Young projectors

$$
\begin{aligned}
& S_{a_{1} a_{2} a_{3}}^{b_{1} b_{2} b_{3}}=\mathbb{P}_{a_{1} a_{2} a_{3}}^{c_{1} c_{2} c_{3}}\left(U_{c_{1}}^{d_{1}} U_{c_{2}}^{d_{2}} U_{c_{3}}^{d_{3}}\right) \mathbb{P}_{d_{1} d_{2} d_{3}}^{S} b_{1} b_{2} b_{3}, \\
& M_{a_{1} a_{2} a_{3}}^{b_{1} b_{2} b_{3}}=\mathbb{P}_{a_{1} a_{2} a_{3}}^{M c_{1} c_{2} c_{3}}\left(U_{c_{1}}^{d_{1}} U_{c_{2}}^{d_{2}} U_{c_{3}}^{d_{3}}\right) \mathbb{P}_{d_{1} d_{2} d_{3}}^{M b_{1} b_{2} b_{3}}, \\
& \tilde{M}_{a_{1} a_{2} a_{3}}^{b_{1} b_{2} b_{3}}=\mathbb{P}_{a_{1} a_{2} a_{3}}^{\tilde{M}} c_{1} c_{2} c_{3}\left(U_{c_{1}}^{d_{1}} U_{c_{2}}^{d_{2}} U_{c_{3}}^{d_{3}}\right) \mathbb{P}_{d_{1} d_{2} d_{3}}^{\tilde{M} b_{1} b_{2} b_{3}},
\end{aligned}
$$

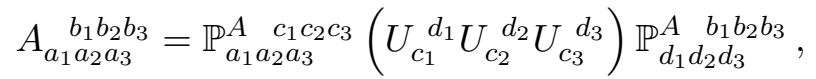

where the Young projectors are given by

$$
\begin{aligned}
\mathbb{P}_{a_{1} a_{2} a_{3}}^{S b_{1} b_{2} b_{3}}= & \frac{1}{6}\left(\delta_{a_{1}}^{b_{1}} \delta_{a_{2}}^{b_{2}} \delta_{a_{3}}^{b_{3}}+\delta_{a_{1}}^{b_{1}} \delta_{a_{2}}^{b_{3}} \delta_{a_{3}}^{b_{2}}+\delta_{a_{1}}^{b_{3}} \delta_{a_{2}}^{b_{1}} \delta_{a_{3}}^{b_{2}}+\delta_{a_{1}}^{b_{3}} \delta_{a_{2}}^{b_{2}} \delta_{a_{3}}^{b_{1}}\right. \\
& \left.+\delta_{a_{1}}^{b_{2}} \delta_{a_{2}}^{b_{3}} \delta_{a_{3}}^{b_{1}}+\delta_{a_{1}}^{b_{2}} \delta_{a_{2}}^{b_{1}} \delta_{a_{3}}^{b_{3}}\right) \\
\mathbb{P}_{a_{1} a_{2} a_{3}}^{M b_{1} b_{2} b_{3}}= & \frac{1}{3}\left(\delta_{a_{1}}^{b_{1}} \delta_{a_{2}}^{b_{2}} \delta_{a_{3}}^{b_{3}}+\delta_{a_{1}}^{b_{2}} \delta_{a_{2}}^{b_{1}} \delta_{a_{3}}^{b_{3}}-\delta_{a_{1}}^{b_{3}} \delta_{a_{2}}^{b_{2}} \delta_{a_{3}}^{b_{1}}-\delta_{a_{1}}^{b_{3}} \delta_{a_{2}}^{b_{1}} \delta_{a_{3}}^{b_{2}}\right) \\
\mathbb{P}_{a_{1} a_{2} a_{3}}^{\tilde{M} b_{1} b_{2} b_{3}}= & \frac{1}{3}\left(\delta_{a_{1}}^{b_{1}} \delta_{a_{2}}^{b_{2}} \delta_{a_{3}}^{b_{3}}+\delta_{a_{1}}^{b_{3}} \delta_{a_{2}}^{b_{2}} \delta_{a_{3}}^{b_{1}}-\delta_{a_{1}}^{b_{2}} \delta_{a_{2}}^{b_{1}} \delta_{a_{3}}^{b_{3}}-\delta_{a_{1}}^{b_{2}} \delta_{a_{2}}^{b_{3}} \delta_{a_{3}}^{b_{1}}\right) \\
\mathbb{P}_{a_{1} a_{2} a_{3}}^{A b_{1} b_{2} b_{3}}= & \frac{1}{6}\left(\delta_{a_{1}}^{b_{1}} \delta_{a_{2}}^{b_{2}} \delta_{a_{3}}^{b_{3}}-\delta_{a_{1}}^{b_{1}} \delta_{a_{2}}^{b_{3}} \delta_{a_{3}}^{b_{2}}+\delta_{a_{1}}^{b_{3}} \delta_{a_{2}}^{b_{1}} \delta_{a_{3}}^{b_{2}}-\delta_{a_{1}}^{b_{3}} \delta_{a_{2}}^{b_{2}} \delta_{a_{3}}^{b_{1}}\right. \\
& \left.+\delta_{a_{1}}^{b_{2}} \delta_{a_{2}}^{b_{3}} \delta_{a_{3}}^{b_{1}}-\delta_{a_{1}}^{b_{2}} \delta_{a_{2}}^{b_{1}} \delta_{a_{3}}^{b_{3}}\right)
\end{aligned}
$$


Using the decomposition (5.17), the integral (5.15) can be written as a sum of integrals of the form (5.6)

$$
\begin{aligned}
I_{3}= & \int_{\mathrm{SU}\left(N_{c}\right)} d U S_{a_{1} a_{2} a_{3}}^{b_{1} b_{2} b_{3}}\left(S^{\dagger}\right) \underset{c_{1} c_{2} c_{3}}{d_{1} d_{2} d_{3}}+\int_{\mathrm{SU}\left(N_{c}\right)} d U A_{a_{1} a_{2} a_{3}}^{b_{1} b_{2} b_{3}}\left(A^{\dagger}\right)_{c_{1} c_{2} c_{3}}^{d_{1} d_{2} d_{3}} \\
& +\int_{\mathrm{SU}\left(N_{c}\right)} d U M_{a_{1} a_{2} a_{3}}^{b_{1} b_{2} b_{3}}\left(M^{\dagger}\right)_{c_{1} c_{2} c_{3}}^{d_{1} d_{2} d_{3}}+\int_{\mathrm{SU}\left(N_{c}\right)} d U \tilde{M}_{a_{1} a_{2} a_{3}}^{b_{1} b_{2} b_{3}}\left(\tilde{M}^{\dagger}\right)_{c_{1} c_{2} c_{3}}^{d_{1} d_{2} d_{3}} \\
& +\int_{\mathrm{SU}\left(N_{c}\right)} d U M_{a_{1} a_{2} a_{3}}^{b_{1} b_{2} b_{3}}\left(\tilde{M}^{\dagger}\right)_{c_{1} c_{2} c_{3}}^{d_{1} d_{2} d_{3}}+\int_{\mathrm{SU}\left(N_{c}\right)} d U \tilde{M}_{a_{1} a_{2} a_{3}}^{b_{1} b_{2} b_{3}}\left(M^{\dagger}\right)_{c_{1} c_{2} c_{3}}^{d_{1} d_{2} d_{3}}
\end{aligned}
$$

where we have not written down the integrals involving representations with different Young tableau shape, as they are zero. The above integrals can be evaluated using the rule (5.6), with the understanding that for the two integrals on the last line, proper care should be taken of the correct placement of the indices. Specifically, in the integral

$$
\int_{\mathrm{SU}\left(N_{c}\right)} d U M_{a_{1} a_{2} a_{3}}^{b_{1} b_{2} b_{3}}\left(\tilde{M}^{\dagger}\right)_{c_{1} c_{2} c_{3}}^{d_{1} d_{2} d_{3}}
$$

the indices $d_{1}, d_{2}, d_{3}$ have the symmetry property indicated by the Young tableau \begin{tabular}{|l|l|}
\hline$d_{1}$ & $d_{3}$ \\
\hline$d_{2}$ &
\end{tabular} of $\tilde{M}$. According to (5.6), they should be distributed on the Young projector corresponding to $M$, i.e. they should be re-ordered such that they have the symmetry property indicated

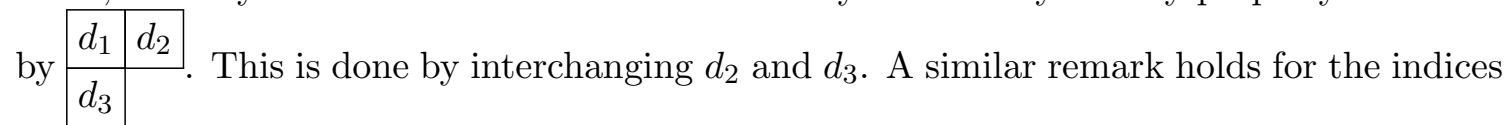
$b_{1}, b_{2}, b_{3}$, so that

$$
\int_{\mathrm{SU}\left(N_{c}\right)} d U M_{a_{1} a_{2} a_{3}}^{b_{1} b_{2} b_{3}}\left(\tilde{M}^{\dagger}\right)_{c_{1} c_{2} c_{3}}^{d_{1} d_{2} d_{3}}=\frac{3}{N_{c}\left(N_{c}^{2}-1\right)} \mathbb{P}_{a_{1} a_{2} a_{3}}^{M d_{1} d_{3} d_{2}} \mathbb{P}_{c_{1} c_{2} c_{3}}^{\tilde{M} b_{1} b_{3} b_{2}}
$$

The last term of (5.20) can be evaluated from analogous considerations. One then finds the following results for the integral (5.15)

$$
\begin{aligned}
I_{3}= & \frac{6}{N_{c}\left(N_{c}+1\right)\left(N_{c}+2\right)} \mathbb{P}_{a_{1} a_{2} a_{3}}^{S d_{1} d_{2} d_{3}} \mathbb{P}_{c_{1} c_{2} c_{3}}^{S b_{1} b_{2} b_{3}}+\frac{3}{N_{c}\left(N_{c}^{2}-1\right)} \mathbb{P}_{a_{1} a_{2} a_{3}}^{M d_{1} d_{2} d_{3}} \mathbb{P}_{c_{1} c_{2} c_{3}}^{M b_{1} b_{2} b_{3}} \\
& +\frac{3}{N_{c}\left(N_{c}^{2}-1\right)} \mathbb{P}_{a_{1} a_{2} a_{3}}^{\tilde{M} d_{1} d_{2} d_{3}} \mathbb{P}_{c_{1} c_{2} c_{3}}^{\tilde{M} b_{1} b_{2} b_{3}}+\frac{3}{N_{c}\left(N_{c}^{2}-1\right)} \mathbb{P}_{a_{1} a_{2} a_{3}}^{M d_{1} d_{3} d_{2}} \mathbb{P}_{c_{1} c_{2} c_{3}}^{\tilde{M} b_{3} b_{3} b_{2}} \\
& +\frac{3}{N_{c}\left(N_{c}^{2}-1\right)} \mathbb{P}_{a_{1} a_{2} a_{3}}^{\tilde{M} d_{1} d_{3} d_{2}} \mathbb{P}_{c_{1} c_{2} c_{3}}^{M b_{1} b_{3} b_{2}}+\frac{6}{N_{c}\left(N_{c}-1\right)\left(N_{c}-2\right)} \mathbb{P}_{a_{1} a_{2} a_{3}}^{A d_{1} d_{2} d_{3}} \mathbb{P}_{c_{1} c_{2} c_{3}}^{A b_{1} b_{2} b_{3}} .
\end{aligned}
$$

The other $I_{m}$ can be calculated in a similar manner. We have given the result for $I_{4}$ in appendix B.

Using the above results, other non-zero integrals can be derived by making use of the $\mathrm{SU}\left(N_{c}\right)$ identities

$$
\begin{aligned}
U_{a_{1}}^{b_{1}} & =\frac{1}{\left(N_{c}-1\right) !} \epsilon_{a_{1} a_{2} \cdots a_{N}} \epsilon^{b_{1} b_{2} \cdots b_{N}}\left(U^{\dagger}\right)_{b_{2}}^{a_{2}} \cdots\left(U^{\dagger}\right)_{b_{N}}^{a_{N}} \\
\left(U^{\dagger}\right)_{a_{1}}^{b_{1}} & =\frac{1}{\left(N_{c}-1\right) !} \epsilon_{a_{1} a_{2} \cdots a_{N}} \epsilon^{b_{1} b_{2} \cdots b_{N}} U_{b_{2}}^{a_{2}} \cdots U_{b_{N}}^{a_{N}}
\end{aligned}
$$


These identities often allow one to reduce group integrals to integrals of the form of $I_{m}$, that can be calculated according to the method outlined above. In this way, one can for instance calculate the baryonic integral

$$
\int_{\mathrm{SU}\left(N_{c}\right)} d U U_{a_{1}}^{b_{1}} \cdots U_{a_{N_{c}}}^{b_{N_{c}}}=\frac{1}{N_{c} !} \epsilon_{a_{1} \cdots a_{N_{c}}} \epsilon^{b_{1} \cdots b_{N_{c}}}
$$

Moreover, the calculation of (5.2) can now be reduced to the calculation of (5.1)

$$
\begin{aligned}
& \int_{\mathrm{SU}(3)} d U U_{a_{1}}^{b_{1}} U_{a_{2}}^{b_{2}} U_{a_{3}}^{b_{3}} U_{a_{4}}^{b_{4}}\left(U^{\dagger}\right)_{c_{1}}^{d_{1}}= \\
& \quad \frac{1}{2} \epsilon_{a_{4} d_{2} d_{3}} \epsilon^{b_{4} c_{2} c_{3}} \int_{\mathrm{SU}(3)} d U U_{a_{1}}^{b_{1}} U_{a_{2}}^{b_{2}} U_{a_{3}}^{b_{3}}\left(U^{\dagger}\right)_{c_{1}}^{d_{1}}\left(U^{\dagger}\right)_{c_{2}}^{d_{2}}\left(U^{\dagger}\right)_{c_{3}}^{d_{3}}, \\
& \int_{\mathrm{SU}(3)} d U U_{a_{1}}^{b_{1}} U_{a_{2}}^{b_{2}} U_{a_{3}}^{b_{3}} U_{a_{4}}^{b_{4}} U_{a_{5}}^{b_{5}} U_{a_{6}}^{b_{6}}= \\
& \quad \frac{1}{4} \epsilon_{a_{5} d_{1} d_{2}} \epsilon^{b_{5} c_{1} c_{2}} \epsilon_{a_{6} d_{3} d_{4}} \epsilon^{b_{6} c_{3} c_{4}} \int_{\mathrm{SU}(3)} d U U_{a_{1}}^{b_{1}} U_{a_{2}}^{b_{2}} U_{a_{3}}^{b_{3}} U_{a_{4}}^{b_{4}}\left(U^{\dagger}\right)_{c_{1}}^{d_{1}}\left(U^{\dagger}\right)_{c_{2}}^{d_{2}}\left(U^{\dagger}\right)_{c_{3}}^{d_{3}}\left(U^{\dagger}\right)_{c_{4}}^{d_{4}}, \\
& \int_{\mathrm{SU}(3)} d U U_{a_{1}}^{b_{1}} U_{a_{2}}^{b_{2}} U_{a_{3}}^{b_{3}} U_{a_{4}}^{b_{4}} U_{a_{5}}^{b_{5}}\left(U^{\dagger}\right)_{c_{1}}^{d_{1}}\left(U^{\dagger}\right)_{c_{2}}^{d_{2}}= \\
& \frac{1}{2} \epsilon_{a_{5} d_{3} d_{4}} \epsilon^{b_{5} c_{3} c_{4}} \int_{\mathrm{SU}(3)} d U U_{a_{1}}^{b_{1}} U_{a_{2}}^{b_{2}} U_{a_{3}}^{b_{3}} U_{a_{4}}^{b_{4}}\left(U^{\dagger}\right)_{c_{1}}^{d_{1}}\left(U^{\dagger}\right)_{c_{2}}^{d_{2}}\left(U^{\dagger}\right)_{c_{3}}^{d_{3}}\left(U^{\dagger}\right)_{c_{4}}^{d_{4}} .
\end{aligned}
$$

Finally, let us note for the sake of completeness that an expression for integrals of the type

$$
\int_{\mathrm{SU}\left(N_{c}\right)} d U U_{a_{1}}^{b_{1}} \cdots U_{a_{N_{c}}}^{b_{N_{c}}} U_{a_{N_{c}+1}}^{b_{N_{c}+1}} \cdots U_{a_{2 N_{c}}}^{b_{2 N_{c}}} \cdots U_{a_{(p-1) N_{c}+1}}^{b_{(p-1) N_{c}+1}} \cdots U_{a_{p N_{c}}}^{b_{p N_{c}}}
$$

is known in terms of $\epsilon$-symbols (see e.g. [15] for a derivation). In particular, the result is given by

$$
\begin{aligned}
& \frac{2 ! \cdot 3 ! \cdots\left(N_{c}-1\right) !}{(p+1) ! \cdots\left(p+N_{c}-1\right) !} \epsilon_{a_{1} \cdots a_{N_{c}}} \epsilon^{b_{1} \cdots b_{N_{c}} \cdots \epsilon_{a_{(p-1) N_{c}+1} \cdots a_{p N_{c}}}} \epsilon^{b_{(p-1) N_{c}+1} \cdots b_{p N_{c}}} \\
& \quad+\text { permutations }
\end{aligned}
$$

where '+ permutations' indicates that one has to add similar terms as the first, where however the indices of the first term are permuted in such a way as to render the resulting expression symmetric under the interchange of all $\left(a_{i}, b_{i}\right)$ index pairs. In principle, one can use this result along with the $\mathrm{SU}\left(N_{c}\right)$ identities (5.24) to calculate the integrals (5.3). One can then rewrite the result in terms of Kronecker-deltas by contracting the various $\epsilon$-symbols and using the identity

$$
\epsilon_{a_{1} \cdots a_{N_{c}}} \epsilon^{b_{1} \cdots b_{N_{c}}}=N_{c} ! \delta_{\left[a_{1}\right.}^{\left[b_{1}\right.} \cdots \delta_{\left.a_{N_{c}}\right]}^{\left.b_{N_{c}}\right]} .
$$

Given the number of permutations one has to add by hand in (5.28), extracting the integrals (5.3) in this way can however be rather cumbersome. 


\subsection{Diagrammatic techniques}

The technique described in the above section is general and can be used to calculate any type of non-zero $\mathrm{SU}\left(N_{c}\right)$ integral. Since a diagram consists of a number of links attached to each other, the group integrals associated to a diagram can be obtained by multiplying the integrals corresponding to the links and by properly contracting their group indices. These contractions can easily be carried out by a symbolic computer program. For simple diagrams, the contractions can also be easily done using diagrammatic techniques explained in reference $[15,17]$, to which we refer for diagrammatic notations and conventions. For example the result for the integral $\int_{\mathrm{SU}\left(N_{c}\right)} d U U_{a}^{b}\left(U^{\dagger}\right)_{c}{ }^{d}$ (given in (5.7)) can be written diagrammatically as

$$
\left.\frac{1}{N_{c}}\right)(
$$

Carefully identifying the links which are connected it is possible to calculate any of the diagrams in section 4 diagrammatically using the appropriate integral equations in section 5 . As a simple example consider the diagram ${ }^{\prime}$ in (4.5). This can be evaluated as

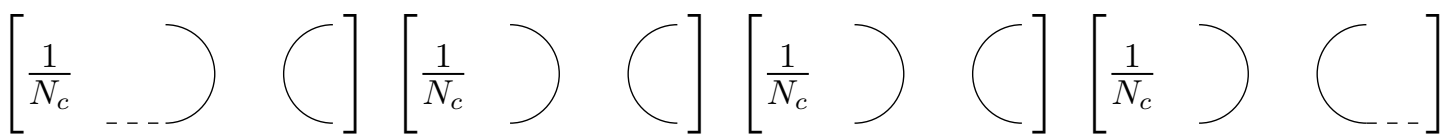

$$
\begin{aligned}
& =\left[\frac{1}{N_{c}}\right]^{4}[\bigcirc \bigcirc \bigcirc \bigcirc \\
& =\left[\frac{1}{N_{c}}\right][\cup] .
\end{aligned}
$$

Similarly the result for $\int_{\mathrm{SU}\left(N_{c}\right)} d U U_{a_{1}}^{b_{1}} U_{a_{2}}^{b_{2}}\left(U^{\dagger}\right)_{c_{1}}^{d_{1}}\left(U^{\dagger}\right)_{c_{2}}^{d_{2}}$ (given in (5.14)) can be written as

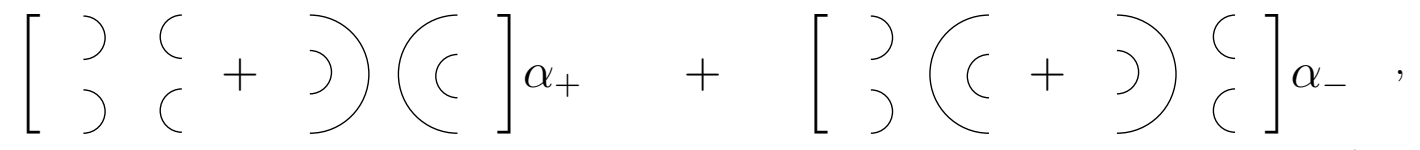

with

$$
\alpha_{ \pm} \equiv \frac{1}{2}\left[\frac{1}{N_{c}\left(N_{c}+1\right)} \pm \frac{1}{N_{c}\left(N_{c}-1\right)}\right]
$$

which can be used to calculate diagrams with four overlapping links, and so on.

Diagrams of one-tile area, that are open in one corner, can also be easily integrated. Since such diagrams have only two free indices, the final result must be given by a constant $C$ times a Kronecker delta for the two indices

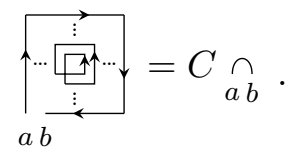


In order to determine the constant $C$ we multiply by $\delta_{a b}$. This leads to a closed diagram. By calculating this closed diagram in two different ways, the constant $C$ can be calculated, as illustrated in the following diagrammatic equation:

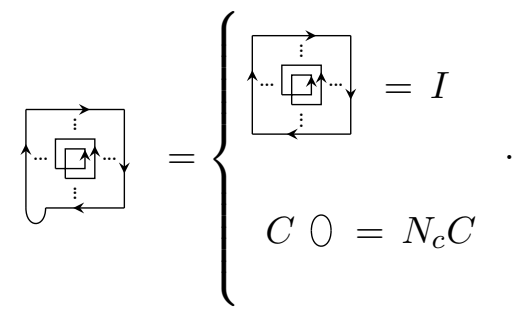

The $I$ in the upper equation on the r.h.s. is the value of the integrated closed diagram, while the lower equation is obtained from (5.34), and using that $\delta_{a a}=N_{c}$ for the fundamental representation. Equating these two different ways of calculating the same diagram, we can thus write

$$
C=\frac{1}{N_{c}} I=\frac{1}{N_{c}} \int_{\mathrm{SU}\left(N_{c}\right)} d U
$$

where the integrand indicated by $\cdots$ depends on the diagram under consideration. Since $I$ corresponds to a closed one-tile diagram, there can be no free indices and the integrand indicated by $\cdots$ is given entirely in terms of traces of powers of $U$ and $U^{\dagger}$. As a rule of thumb that can be used to write this integrand down, one can use that for every loop in the diagram that winds around $n$ times in one direction, one should include a factor of $\operatorname{tr} U^{n}$ in the integrand. Likewise a factor of $\operatorname{tr} U^{\dagger^{n}}$ should be included in the integrand for every loop that winds around $n$ times in the other direction. These one-tile closed diagram integrals can then be evaluated very easily using the Young projector formulas of the previous section, or using the diagrammatic techniques of [15]. In this way, the calculation of this type of diagrams can be reduced to calculating a single group integral, instead of calculating four group integrals (one for every link) and multiplying and contracting the results.

As an illustrative example we calculate the value of the diagram

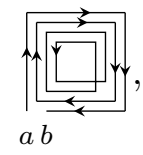

where the corresponding closed diagram is

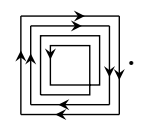

Using equations (5.34) and (5.36) the open diagram evaluates to

$$
\underset{a b}{\square}=\frac{\delta_{a b}}{N_{c}} \int_{\mathrm{SU}\left(N_{c}\right)} d U \operatorname{tr}^{2} U^{\dagger} \operatorname{tr} U^{2}
$$


where the integral corresponds to the value of the closed diagram (5.38). The integrand is determined using the above stated rule of thumb, by noting that the closed diagram consists of three loops: the outer two winding one time in one direction, while the inner loop winds two times in the other direction. This integral can be very easily evaluated using e.g. the Young projector formula (5.14) as

$$
\int_{\mathrm{SU}\left(N_{c}\right)} d U \operatorname{tr}^{2} U^{\dagger} \operatorname{tr} U^{2}=\int_{\mathrm{SU}\left(N_{c}\right)} d U U_{a_{1}}^{b_{1}} U_{b_{1}}^{a_{1}}\left(U^{\dagger}\right)_{c_{1}}^{c_{1}}\left(U^{\dagger}\right)_{c_{2}}^{c_{2}}=0
$$

where the last equation is obtained by plugging the indices in in (5.14) and evaluating the resulting formula explicitly. We thus find that the diagram (5.37) evaluates to zero.

\section{Sources of error}

\subsection{Mis-counting of overlapping graphs}

One of the potentially problematic aspects of our approach is that since each diagram type can be placed at a site $x$, any number of times, in any possible direction, over-counting will result from contributions with overlapping diagrams. ${ }^{5}$ This is a problem which arises when non-tree diagrams are included due to the link integrations. It is in principle possible to systematically account for mis-counted graphs order by order by adding the appropriate counter term. However, practically speaking, it is difficult to do this within the formulation we are using. Here are some examples of mis-counted overlapping graphs.

\subsection{1 $L=8$}

$$
=\frac{1}{2 !}\left(-\frac{1}{4 m^{2}}\right)^{8}(-1)^{4}\left(-N_{f}\right)^{2}[0]
$$

however, it gets counted as

$$
\left(-\frac{1}{4 m^{2}}\right)^{8}(-1)^{4}\left(-N_{f}\right)^{2}\left[\frac{1}{N_{c}^{2}}\right]
$$

To account for the above mis-counting, it is necessary to add a counter term at $L=8$ of the form

$$
\prod_{\text {c.t. }}=-\left(-\frac{1}{4 m^{2}}\right)^{8}(-1)^{4}\left(-N_{f}\right)^{2}\left[\frac{1}{N_{c}^{2}}\right]\{4 d(d-1)\}
$$

\footnotetext{
${ }^{5}$ We note that overlapping diagrams are not mis-counted when including only $a$-type contributions, as in the $N_{f}=0$ calculations $[8,9]$.
} 


\subsection{2 $L=12$}

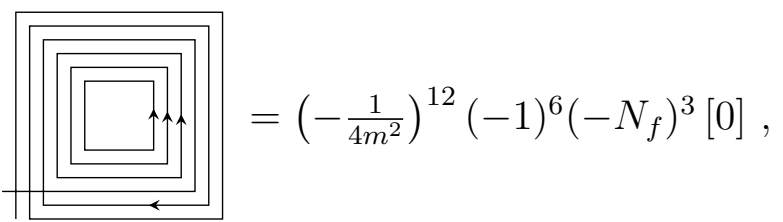

for $N_{c} \geq 3$. For $N_{c}=2$ the result is $\left(-\frac{1}{4 m^{2}}\right)^{12}(-1)^{6}\left(-N_{f}\right)^{3}\left[-\frac{1}{2}\right]$. However, in either case it gets counted as

$$
\left(-\frac{1}{4 m^{2}}\right)^{12}(-1)^{6}\left(-N_{f}\right)^{3}\left[\frac{1}{N_{c}^{3}}\right]
$$

The difficulties in adding counter terms are 1.) it is difficult to determine where exactly to add them within our formulation, and 2.) the counter terms lead to mis-counting at higher orders, requiring the addition of even more counter terms. Since the second issue can be resolved order by order, the first issue is the most critical. If one naively adds the counter term (6.3) as a base diagram at order $L=8$, then indeed the wrong contributions obtained with two overlapping $b$-type diagrams can be cancelled off. However, in addition, new diagrams would be created with both contributions from overlapping $b$-type diagrams, and counter terms of the form (6.3). These mixed diagrams should not be included and would introduce a different, difficult to quantify source of error. Therefore, at this point, we don't attempt to correct for errors resulting from overlapping diagrams. A proper treatment of the issue of overlapping diagrams is left for future research.

\subsection{Avoiding over-counting of graphs}

Another source of error results from over-counting or under-counting of graphs. This happens, for example, when attaching a trunk, $\uparrow$ ( $a$-type), to either of two adjacent corners of a box, $\$$ (b-type) diagram. This results in graphs of the form [12]

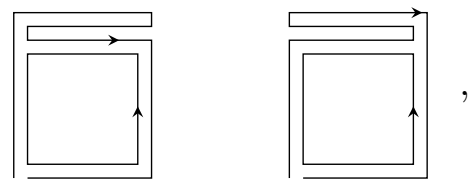

which are identical since the same sequence of links, $U_{\nu}(x) U_{\mu}(x+\hat{\nu}) U_{\mu}^{\dagger}(x+\hat{\nu}) U_{\mu}(x+$ $\hat{\nu}) U_{\nu}^{\dagger}(x+\hat{\mu}) U_{\mu}^{\dagger}(x)$ (outside, plus inside plaquette), appears in both diagrams. To deal with this issue we follow [12] and subtract off one possible direction when attaching a trunk ( $a$ type) to a box (b-type) diagram. At one corner it is necessary to subtract off two directions to avoid over-counting either of
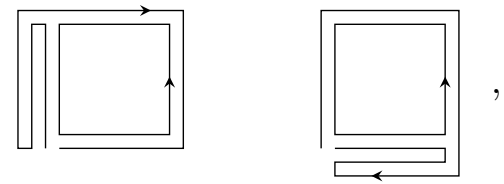
which also appear by attaching both an $a$-type and $b$-type diagram directly at $x$ (when irreducible diagrams are combined). That is, they correspond to
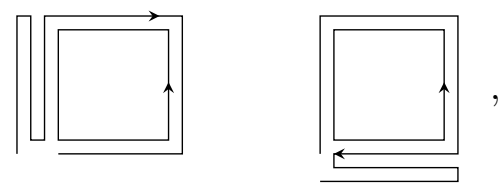

respectively. This result can be generalised for attachment of an $a$-type diagram to any area 1-type diagram. Therefore, the dimensionalities are $a_{1}=2 d-1, a_{1}^{\prime}=2(d-1)$, where $a_{1}^{\prime}$ corresponds to attachment at one (outer) corner of an area 1 diagram, and $a_{1}$ corresponds to attachment at any of the other 6 possible locations. For example, one can choose the outer corner farthest from $x$,

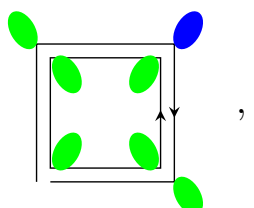

where the blue leaf corresponds to an $a_{1}^{\prime}$ attachment site and the green leaves correspond to an $a_{1}$ attachment site.

\subsubsection{Overlapping of $b$-type graphs}

In the calculation of the dimensionality for attaching b-type graphs one can also make improvements by removing contributions which lead to over-counting. One example results from allowing $b$-type diagrams to overlap. For example,

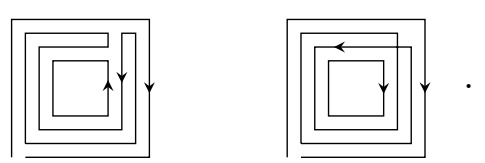

The first graph is already counted as it corresponds to

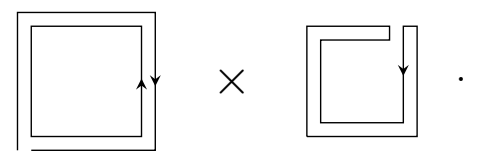

Since it factorises into a separately integrable contribution from the correlator (left) and a contribution from the determinant (right), the contribution from the determinant cancels against the denominator, resulting in a contribution already contained in

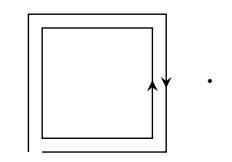

The second graph is not already included so one could allow for it. However, performing the group integrations, the contribution from this graph is

$$
\text { W }
$$


Since this graph would be counted incorrectly by multiplying the separate contributions of the two $b$-type graphs we should disallow it as well. The same arguments can be used to justify disallowing overlapping $b$-type diagrams of the form

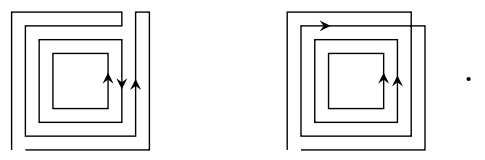

Allowing $b$-type graphs to overlap as in the first diagram would result in over-counting due to factorisation. Allowing them to overlap as in the second diagram would also result in mis-counting, since the diagram evaluates to zero.

\subsubsection{Avoiding over-counting of $b$-type graphs}

To improve the dimensionality $b_{1}$, for attaching $b$-type graphs to area 1 type graphs, it is useful to subtract off dimensions which lead to over-counting. For example, attaching a $b$-type graph to the leaf in

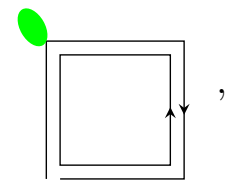

could result (among others) in diagrams of the form
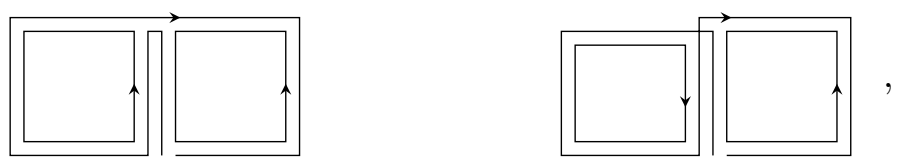

which would lead to over-counting. The first diagram corresponds to attaching

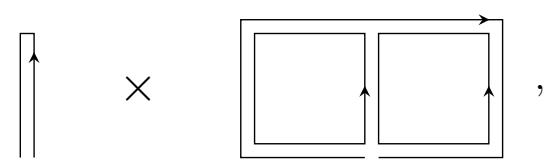

at $x$. The second corresponds to

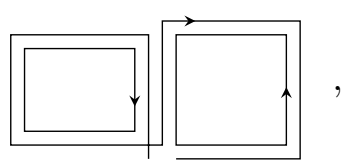

which is formed by combining two $b$-type diagrams at $x$. Avoiding also direct overlap of $b$-type diagrams discussed in the previous subsection, the dimensionality at the external corners neighbouring $x$ is $b_{1}=4(d-1)^{2}$.

Consider the addition of a $b$-type diagram at one of the internal corners

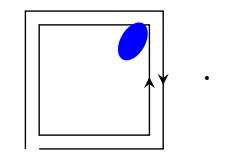


One possible attachment would look like

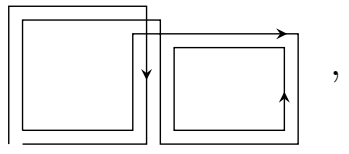

however, this one is equivalent to

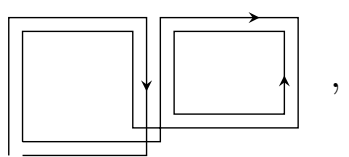

where the attachment is at the lower right internal corner. It is therefore important, when attaching a neighbouring area 1-diagram, to remove the contributions to the dimensionality from re-tracing along the internal plaquette. There are $2(d-1)$ ways to attach in this way from one of the internal corners, and we need to remove an additional contribution from direct overlap of area 1-diagrams by backtracking along a link. The remaining contribution is $b_{1}^{\prime}=4 d(d-1)-[2(d-1)+1]$.

Finally consider attachment to the far external corner

One possible attachment is

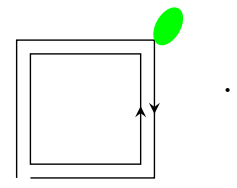

which is equivalent to
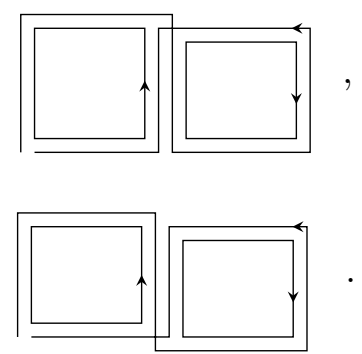

Including all possible ways of folding the diagram which would lead to double counting, the contribution to subtract off the dimensionality is $2(d-1)$. Since an area-1 diagram can also neighbour the top link in the same way this amount needs to be subtracted twice. The total dimensionality at the external corner is therefore $b_{1}=4(d-1)^{2}$.

\subsection{Over-counting resulting from symmetries}

In this section we examine diagrams with symmetries. In the first case, this symmetry leads to over-counting, and in the second case it does not.

Consider a graph of the form (4.10),

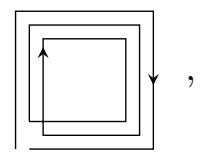

which contains a gauge field loop that winds twice before closing on itself. The graphs in (4.15), (4.16) also belong to this category. One source of over-counting comes about 
when asymmetric attachments are made to the multiply-wound loop. In this case, the overcounting results due to symmetry under rotations by 4 lattice sites of the internal loop. For example, consider two different attachments, represented by the green and blue leaves in

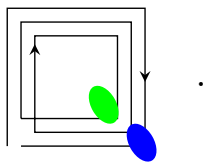

Since these are both attached to the same loop in the same corner it makes no difference if one attaches at the green leaf or the blue one. Such attachments result in identical diagrams which can be transformed into each other under rotations by 4 lattice sites. Therefore if attachment at both sites is allowed with the same dimensionality then there will be over-counting. Over-counting also results when the attachments are made in two different corners on the same loop, since the attachments can be shifted 4 sites along the loop to give an identical diagram, which gets counted separately. Notice that, if identical attachments are made at both the green and blue attachment sites simultaneously then there is no over-counting. We have not yet accounted for this effect in our calculations, so it is a source of error.

It is important to note that not all symmetries lead to over-counting. There also exists a symmetry in diagrams of the form

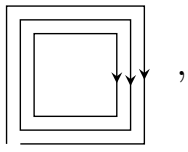

with respect to interchange of the two internal loops (also true in $L=8$ diagrams of the form (4.14)). In this case there is no over-counting when making asymmetric attachments to the internal loops. The contributions from the internal loops come down from the exponential in (2.7), so if one of the loops takes a different shape, then it is necessary to count it twice.

\section{Results}

Using the procedure outlined in section 3, and the considerations outlined in the previous section for reducing over-counting, it is possible to obtain the chiral condensate to some order by solving the appropriate truncated system of equations. In what follows we present results including area 0 and 1 diagrams up to order $L=8$.

\subsection{Asymptotic solutions for large $N_{f} / N_{c}$}

First consider the contributions up to $L=4$, that is all possible diagrams formed from type $a$ (4.4) and type $b$ (4.5) sub-diagrams. The system of equations, using (3.19), (3.20), and the considerations in section 6.2 , is

$$
g_{a}=\frac{1}{a_{0} g_{a}+b_{0} g_{b}},
$$




$$
g_{b}=\frac{\frac{N_{f}}{N_{c}}}{\left(a_{1} g_{a}+b_{1}^{\prime} g_{b}\right)^{4}\left(a_{1} g_{a}+b_{1} g_{b}\right)^{2}\left(a_{1}^{\prime} g_{a}+b_{1} g_{b}\right)},
$$

where the dimensionalities $x_{n}$ are given in appendix A. The chiral condensate as a function of $N_{f}$ can be obtained from (3.18) and (3.23).

We are interested in finding real roots of the set of self-consistent equations for large $N_{f} / N_{c}$, where we will take $d=4$ in what follows. Solving (7.1) for $g_{b}$ and plugging this solution in (7.2), we find that solutions for $g_{a}$ are determined by the roots of the polynomial equation

$$
\begin{aligned}
& \frac{2825761}{10504375}-\frac{32255028}{10504375} g_{a}^{2}+\frac{15618171}{1500625} g_{a}^{4}-\frac{707824}{42875} g_{a}^{6}+\left(\frac{2403}{175}-\frac{60466176}{10504375} \frac{N_{f}}{N_{c}}\right) g_{a}^{8} \\
& \quad-\frac{204}{35} g_{a}^{10}+g_{a}^{12}=0 .
\end{aligned}
$$

Once real solutions for $g_{a}$ of the above polynomial have been found, the corresponding real solutions for $g_{b}$ are found from (7.1)

$$
g_{b}=\frac{1}{b_{0} g_{a}}-\frac{a_{0}}{b_{0}} g_{a} .
$$

The number of real roots of (7.3) in a certain interval can be found by applying Sturm's theorem. For generic ${ }^{6}$ values of $N_{f} / N_{c}$, one finds that the number of real roots in the interval $(0,+\infty)$ is given by 2 . Since the polynomial $(7.3)$ is even in $g_{a}$, the negatives of these roots are also roots and hence there are four real roots in total.

Here, we are interested in finding asymptotic expansions for the roots of (7.3), for large $N_{f} / N_{c} \gg 1$. Multiplying (7.3) by $\epsilon=N_{c} / N_{f}$, we wish to apply perturbation theory to obtain real solutions of

$$
\begin{aligned}
& \epsilon \frac{2825761}{10504375}-\epsilon \frac{32255028}{10504375} g_{a}^{2}+\epsilon \frac{15618171}{1500625} g_{a}^{4}-\epsilon \frac{707824}{42875} g_{a}^{6}+\left(\epsilon \frac{2403}{175}-\frac{60466176}{10504375}\right) g_{a}^{8} \\
& -\epsilon \frac{204}{35} g_{a}^{10}+\epsilon g_{a}^{12}=0,
\end{aligned}
$$

for $\epsilon \ll 1$. Asymptotic expansions in $\epsilon$ for the roots of this polynomial can then be found via singular perturbation theory $[18,19]$. In particular, one looks for roots of the form

$$
g_{a}=\epsilon^{P} w(\epsilon),
$$

where $w(\epsilon)$ is regular in the limit $\epsilon \rightarrow 0$ and $\lim _{\epsilon \rightarrow 0} w(\epsilon)$ is assumed to be non-zero. The exponent $P$ can be determined via singular perturbation theory to be either $-1 / 4$ or $1 / 8$. Let us focus on solutions with $P=-1 / 4$ first. Plugging $g_{a}=\epsilon^{-1 / 4} w(\epsilon)$ in (7.5), one obtains

$$
\begin{gathered}
\frac{2825761}{10504375} \epsilon^{3}-\frac{32255028}{10504375} \epsilon^{5 / 2} w(\epsilon)^{2}+\frac{15618171}{1500625} \epsilon^{2} w(\epsilon)^{4}-\frac{707824}{42875} \epsilon^{3 / 2} w(\epsilon)^{6} \\
-\frac{60466176}{10504375} w(\epsilon)^{8}+\frac{2403}{175} \epsilon w(\epsilon)^{8}-\frac{204}{35} \sqrt{\epsilon} w(\epsilon)^{10}+w(\epsilon)^{12}=0 .
\end{gathered}
$$

\footnotetext{
${ }^{6}$ For very small values of $N_{f} / N_{c}\left(N_{f} / N_{c}<2 \cdot 10^{-6}\right)$, the polynomial (7.3) has four real roots.
} 
Upon renaming $\epsilon=\beta^{2}$, one obtains an expression that only involves integer powers of $\beta$

$$
\begin{gathered}
\frac{2825761}{10504375} \beta^{6}-\frac{32255028}{10504375} \beta^{5} w(\beta)^{2}+\frac{15618171}{1500625} \beta^{4} w(\beta)^{4}-\frac{707824}{42875} \beta^{3} w(\beta)^{6} \\
-\frac{60466176}{10504375} w(\beta)^{8}+\frac{2403}{175} \beta^{2} w(\beta)^{8}-\frac{204}{35} \beta w(\beta)^{10}+w(\beta)^{12}=0 .
\end{gathered}
$$

One can then propose an ordinary series solution for $w(\beta)$

$$
w(\beta)=\sum_{i=0}^{\infty} \omega_{i} \beta^{i} .
$$

The coefficients $\omega_{i}$ can be solved for by plugging (7.9) in (7.8) and requiring that the result is zero at every order in $\beta$. This leads to a set of equations for $\omega_{i}$, that can be solved in an iterative manner. Restricting ourselves to sixth order in $\beta$, we thus obtain asymptotic expansions for two solutions, that are each others negatives. Expressed again in terms of $\epsilon$, these are given by

$$
\begin{aligned}
g_{a}= \pm & \frac{1}{\epsilon^{1 / 4}}\left(\frac{36 \sqrt{6}}{357^{1 / 4}}+\frac{177^{1 / 4} \sqrt{\epsilon}}{12 \sqrt{6}}-\frac{451857^{3 / 4} \epsilon}{124416 \sqrt{6}}+\frac{111508699757^{1 / 4} \epsilon^{3 / 2}}{8707129344 \sqrt{6}}\right. \\
& -\frac{1451051387931257^{3 / 4} \epsilon^{2}}{180551034077184 \sqrt{6}}+\frac{6160800114024631257^{1 / 4} \epsilon^{5 / 2}}{155996093442686976 \sqrt{6}} \\
& \left.-\frac{432151205059302107093757^{3 / 4} \epsilon^{3}}{14556307471324007104512 \sqrt{6}}\right) .
\end{aligned}
$$

Similarly, for $P=1 / 8$, one obtains asymptotic expansions for two solutions, that are each others negatives and are given by

$$
\begin{aligned}
g_{a}= \pm & \epsilon^{1 / 8}\left(\frac{\sqrt{41}}{66^{1 / 4}}-\frac{13 \sqrt{41} \epsilon^{1 / 4}}{486^{3 / 4}}+\frac{3995 \sqrt{41} \sqrt{\epsilon}}{622086^{1 / 4}}-\frac{2827435 \sqrt{41} \epsilon^{3 / 4}}{134369286^{3 / 4}}+\frac{85021433 \sqrt{41} \epsilon}{8599633926^{1 / 4}}\right. \\
& \left.-\frac{141672440399 \sqrt{41} \epsilon^{5 / 4}}{5572562780166^{3 / 4}}+\frac{13186932605159 \sqrt{41} \epsilon^{3 / 2}}{802449040343046^{1 / 4}}\right) .
\end{aligned}
$$

Asymptotic expansions for $g_{b}$ can then be found by using these expansions for $g_{a}$ in (7.4). The expansions for $g_{a}$ and $g_{b}$ can be used to obtain approximate solutions for the chiral condensate, that are valid for $N_{f} / N_{c} \gg 1$. In particular, one obtains two positive solutions for the chiral condensate $\lim _{m \rightarrow 0} \operatorname{tr}[G(x, x)] /\left(N_{s} N_{f} N_{c}\right)=2 / g$, given by

$$
\begin{aligned}
\frac{2}{g}= & \frac{35 \cdot 7^{1 / 4}}{24 \sqrt{6}}\left(\frac{N_{c}}{N_{f}}\right)^{1 / 4}-\frac{1225 \cdot 7^{3 / 4}}{11664 \sqrt{6}}\left(\frac{N_{c}}{N_{f}}\right)^{3 / 4}+\frac{1651587875 \cdot 7^{1 / 4}}{5804752896 \sqrt{6}}\left(\frac{N_{c}}{N_{f}}\right)^{5 / 4} \\
& -\frac{1810166421875 \cdot 7^{3 / 4}}{11284439629824 \sqrt{6}}\left(\frac{N_{c}}{N_{f}}\right)^{7 / 4}+\frac{2087791584389809375 \cdot 7^{1 / 4}}{2807929681968365568 \sqrt{6}}\left(\frac{N_{c}}{N_{f}}\right)^{9 / 4} \\
& -\frac{163362753019994171875 \cdot 7^{3 / 4}}{303256405652583481344 \sqrt{6}}\left(\frac{N_{c}}{N_{f}}\right)^{11 / 4}+\mathcal{O}\left(\left(N_{c} / N_{f}\right)^{13 / 4}\right)
\end{aligned}
$$



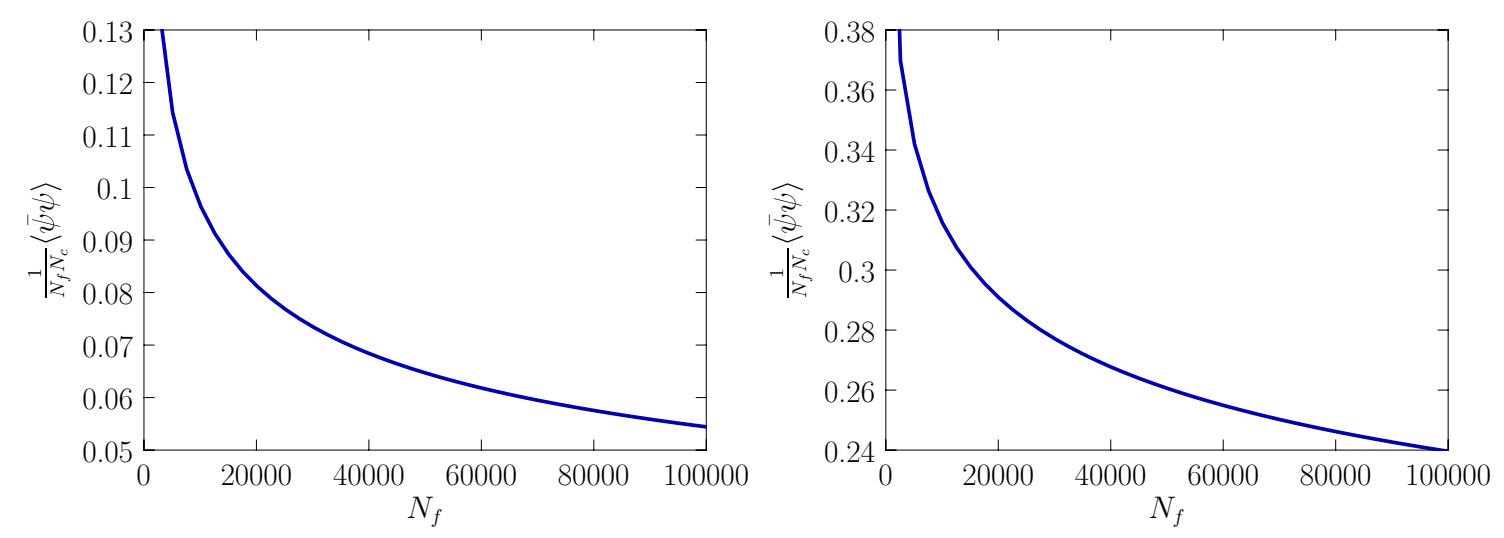

Figure 1. Plots of the two (positive) approximate solutions for the chiral condensate, for large $N_{f} / N_{c}$. The left figure represents the solution of (7.12), the right figure the solution of (7.13).

and

$$
\begin{aligned}
\frac{2}{g}= & \frac{\sqrt{41}}{4 \cdot 6^{1 / 4}}\left(\frac{N_{c}}{N_{f}}\right)^{1 / 8}-\frac{35 \sqrt{41}}{288 \cdot 6^{3 / 4}}\left(\frac{N_{c}}{N_{f}}\right)^{3 / 8}-\frac{10073 \sqrt{41}}{124416 \cdot 6^{1 / 4}}\left(\frac{N_{c}}{N_{f}}\right)^{5 / 8} \\
& +\frac{35399 \sqrt{41}}{2985984 \cdot 6^{3 / 4}}\left(\frac{N_{c}}{N_{f}}\right)^{7 / 8}-\frac{103461197 \sqrt{41}}{15479341056 \cdot 6^{1 / 4}}\left(\frac{N_{c}}{N_{f}}\right)^{9 / 8} \\
& +\frac{171638444453 \cdot \sqrt{41}}{1114512556032 \cdot 6^{3 / 4}}\left(\frac{N_{c}}{N_{f}}\right)^{11 / 8}+\mathcal{O}\left(\left(N_{c} / N_{f}\right)^{13 / 8}\right) .
\end{aligned}
$$

These two solutions are plotted (for large $N_{f} / N_{c}$ ) in figure 1 .

\subsection{Numerical results for $N_{c}=3$}

Consider again the contributions up to $L=4$, formed from type $a$ (4.4) and type $b$ (4.5) sub-diagrams. The system of equations for $g_{a}$ and $g_{b}$ is as in the previous subsection given by (7.1), (7.2), including the considerations in section 6.2 , such that the chiral condensate as a function of $N_{f}$ can be obtained from (3.18) and (3.23) by solving the system of equations numerically.

Results for $\frac{1}{N_{f} N_{c}}\langle\bar{\psi} \psi\rangle$, including base diagrams up to $L=4$, with $N_{c}=3$ and $d=4$ are shown in figure 2 (left). As in the previous section, solving the system of equations results in two solutions. One of these, solution 1, approaches the result of [7, 9] as $N_{f} \rightarrow 0$. For the other, solution $2, \frac{1}{N_{f} N_{c}}\langle\bar{\psi} \psi\rangle \rightarrow \infty$ as $N_{f} \rightarrow 0$. In the limit $N_{f} \rightarrow \infty$, both solutions approach zero, solution 2 falling off more quickly. There is no sign of a discontinuity, at any $N_{f}$, for either of the solutions.

To determine the effect of including higher order diagrams consider the contributions of area 1 diagrams up to $L=6$, formed from type $a, b$, and $d(4.7)-(4.10)$ sub-diagrams. The system of equations is

$$
\begin{aligned}
g_{a} & =\frac{1}{a_{0} g_{a}+b_{0} g_{b}+b_{0} g_{d}}, \\
g_{b} & =\frac{\frac{N_{f}}{N_{c}}}{\left(a_{1} g_{a}+b_{1}^{\prime} g_{b}+b_{1}^{\prime} g_{d}\right)^{4}\left(a_{1} g_{a}+b_{1} g_{b}+b_{1} g_{d}\right)^{2}\left(a_{1}^{\prime} g_{a}+b_{1} g_{b}+b_{1} g_{d}\right)},
\end{aligned}
$$



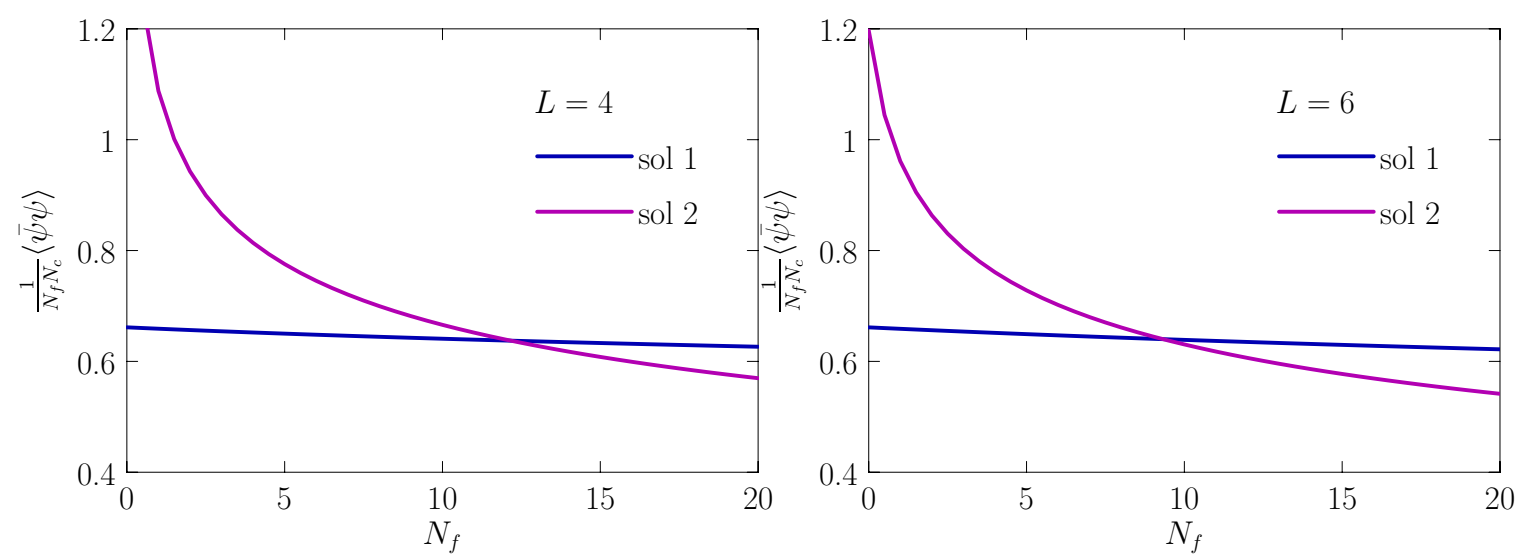

Figure 2. $\frac{1}{N_{f} N_{c}}\langle\bar{\psi} \psi\rangle$ vs. $N_{f}$ including area 1 diagrams up to order $L=4$ (left), and $L=6$ (right).

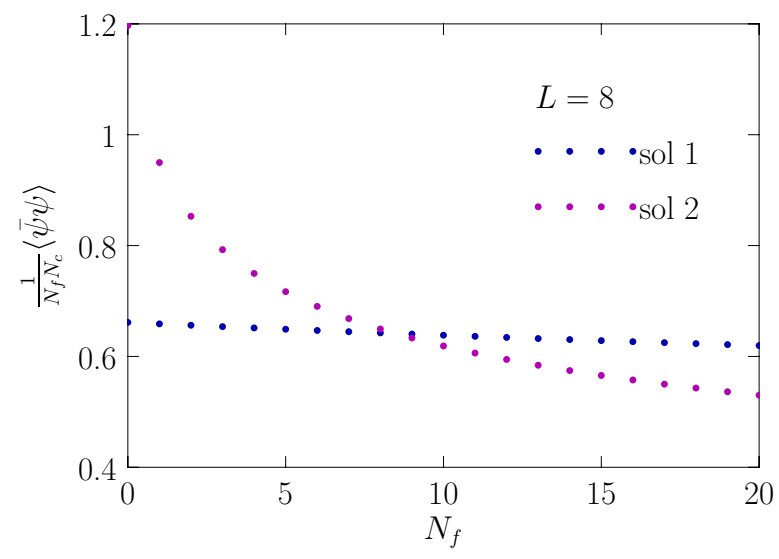

Figure 3. $\frac{1}{N_{f} N_{c}}\langle\bar{\psi} \psi\rangle$ vs. $N_{f}$ including area 1 diagrams up to order $L=8$ (left).

$$
g_{d}=\frac{\frac{1}{3}\left(\frac{1}{2} N_{f}^{2}+2 N_{f}+1\right)}{\left(a_{1} g_{a}+b_{1}^{\prime} g_{b}+b_{1}^{\prime} g_{d}\right)^{8}\left(a_{1} g_{a}+b_{1} g_{b}+b_{1} g_{d}\right)^{2}\left(a_{1}^{\prime} g_{a}+b_{1} g_{b}+b_{1} g_{d}\right)} .
$$

In (7.16), we have explicitly set $N_{c}=3$, as the contribution from $d$-type diagrams is otherwise zero. The results for $\frac{1}{N_{f} N_{c}}\langle\bar{\psi} \psi\rangle$ from (7.14)-(7.16) as a function of $N_{f}$ (and with $N_{c}=3$ ) are shown in figure 2 (right). The results are quite similar to the case of $L=4$, suggesting that the solutions are converging, however, solution 2 now approaches a finite value around 1.2 in the limit $N_{f} \rightarrow 0$. For all $N_{f}$, the values of $\frac{1}{N_{f} N_{c}}\langle\bar{\psi} \psi\rangle$ have decreased. In the limit $N_{f} \rightarrow \infty$, the differences from the $L=4$ truncation become more apparent but both solutions still approach zero, without exhibiting any discontinuities.

Finally, consider the effect of including contributions of area 1 diagrams up to $L=8$, formed from type $a, b, d$, and $g$ (4.14)-(4.16) sub-diagrams. The system of equations is

$g_{a}=\frac{1}{a_{0} g_{a}+b_{0} g_{b}+b_{0} g_{d}+b_{0} g_{g}}$, 

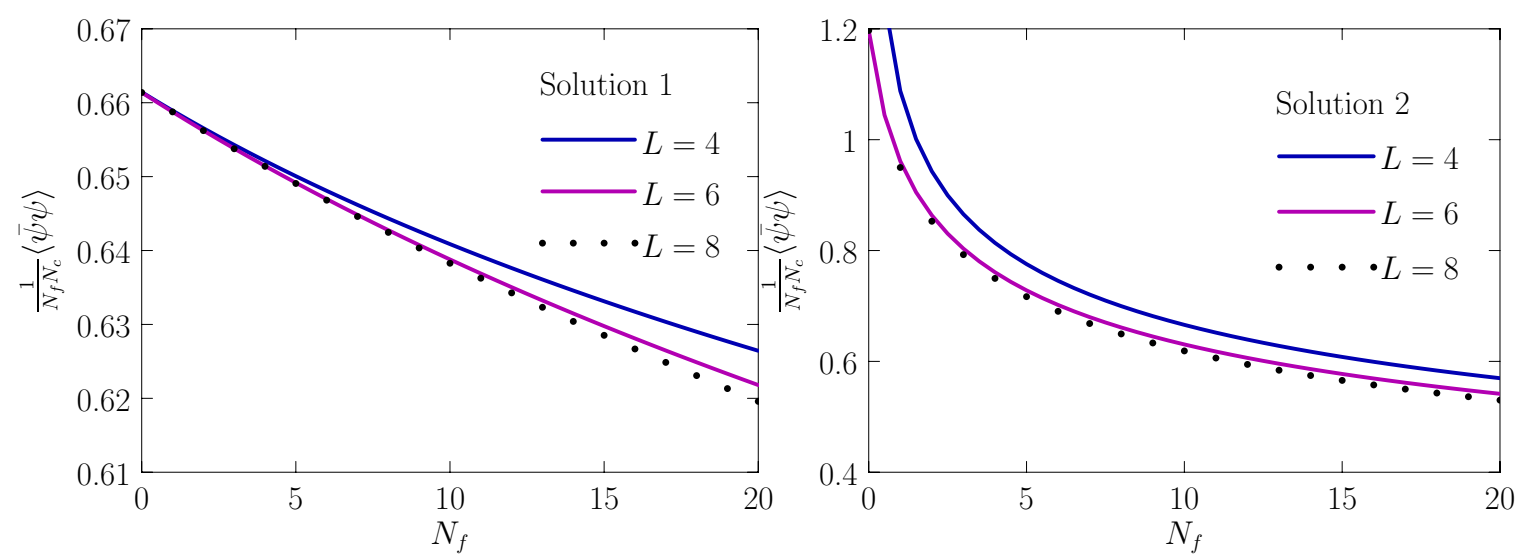

Figure 4. $\frac{1}{N_{f} N_{c}}\langle\bar{\psi} \psi\rangle$ vs. $N_{f}$ including area 1 diagrams up to order $L=4,6,8$ for solution 1 (left), and solution 2 (right).

$$
\begin{aligned}
& g_{b}=\frac{\frac{N_{f}}{N_{c}}}{\left(a_{1} g_{a}+b_{1}^{\prime} g_{b}+b_{1}^{\prime} g_{d}+b_{1}^{\prime} g_{g}\right)^{4}\left(a_{1} g_{a}+b_{1} g_{b}+b_{1} g_{d}+b_{1} g_{g}\right)^{2}\left(a_{1}^{\prime} g_{a}+b_{1} g_{b}+b_{1} g_{d}+b_{1} g_{g}\right)}, \\
& g_{d}=\frac{\frac{1}{3}\left(\frac{1}{2} N_{f}^{2}+2 N_{f}+1\right)}{\left(a_{1} g_{a}+b_{1}^{\prime} g_{b}+b_{1}^{\prime} g_{d}+b_{1}^{\prime} g_{g}\right)^{8}\left(a_{1} g_{a}+b_{1} g_{b}+b_{1} g_{d}+b_{1} g_{g}\right)^{2}\left(a_{1}^{\prime} g_{a}+b_{1} g_{b}+b_{1} g_{d}+b_{1} g_{g}\right)} \\
& g_{g}=\frac{\frac{1}{N_{c}}\left(N_{f}^{3}+2 N_{f}\right)}{\left(a_{1} g_{a}+b_{1}^{\prime} g_{b}+b_{1}^{\prime} g_{d}+b_{1}^{\prime} g_{g}\right)^{12}\left(a_{1} g_{a}+b_{1} g_{b}+b_{1} g_{d}+b_{1} g_{g}\right)^{2}\left(a_{1}^{\prime} g_{a}+b_{1} g_{b}+b_{1} g_{d}+b_{1} g_{g}\right)}
\end{aligned}
$$

The results for $\frac{1}{N_{f} N_{c}}\langle\bar{\psi} \psi\rangle$ as a function of $N_{f}$ are given in figure 3 . While the data points haven't shifted so much from the $L=6$ results, one notable difference is the absence of real solutions for $\frac{1}{N_{f} N_{c}}\langle\bar{\psi} \psi\rangle$ for non-integer values of $N_{f}$. The reason for this is unclear. At lower orders, the two real solutions were continuous solutions for all $N_{f}$.

The results for each solution of $\frac{1}{N_{f} N_{c}}\langle\bar{\psi} \psi\rangle$ as a function of $N_{f}$ from (7.17)-(7.20) for the $L=8$ truncation are reproduced in figure 4 , along with those from the $L=6$ truncation in (7.14)-(7.16), and from the $L=4$ truncation in (7.1)-(7.2), showing how the solutions change as a function of the truncation order $L$. In both cases the solution appears to be converging, at least for the smaller values of $N_{f}$.

Finally, to check convergence, the values of each contribution $d_{b} g_{b}, d_{d} g_{d}, d_{g} g_{g}$ to (3.18), that solve the system of equations in (7.17)-(7.20), are plotted in figure 5. While in both solutions the higher order contributions from $d_{d} g_{d}$ and $d_{g} g_{g}$ are smaller in magnitude, these contributions have the potential to become more significant at larger values of $N_{f}$, since $g_{d}$ goes like $N_{f}^{2}(7.19)$, and $g_{g}$ goes like $N_{f}^{3}(7.20)$.

\subsection{Comparison with lattice data}

In order to test the plausibility of our results we perform calculations of $\frac{1}{N_{f}}\langle\bar{\psi} \psi\rangle$ as a function of the quark mass $m a$, in order to compare with presently available lattice data [13]. 


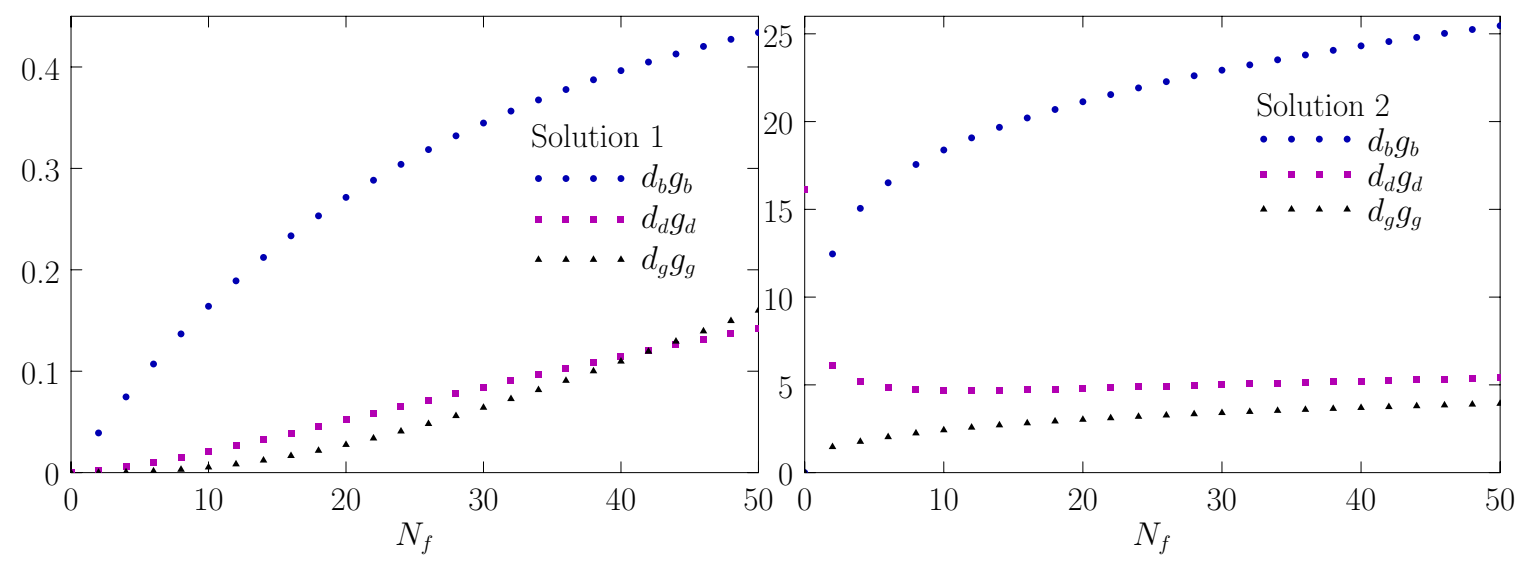

Figure 5. $g_{x}$ vs. $N_{f}$ including area 1 diagrams up to order $L=8$ for solution 1 (left), and solution 2 (right).

\begin{tabular}{|l|l|l|l|l|}
\hline$m a$ & $L=4$ & $L=6$ & $L=8$ & $\mathrm{MC}[13]$ \\
\hline 0.025 & 1.9703 & 1.9701 & 1.9701 & $1.9710(11)$ \\
\hline 0.05 & 1.9562 & 1.9561 & 1.9561 & $1.9574(7)$ \\
\hline 0.1 & 1.9283 & 1.9282 & 1.9282 & $1.9295(5)$ \\
\hline
\end{tabular}

Table 1. $\frac{1}{N_{f}}\langle\bar{\psi} \psi\rangle$ with $N_{f}=0$ for various ma including area 0 and 1 subdiagrams up to $L=4,6,8$.

\begin{tabular}{|l|l|l|l|l|}
\hline$m a$ & $L=4$ & $L=6$ & $L=8$ & $\mathrm{MC}[13]$ \\
\hline 0.025 & 1.9442 & 1.9424 & 1.9422 & $1.9520(6)$ \\
\hline 0.05 & 1.932 & 1.9303 & 1.9302 & $1.9413(3)$ \\
\hline 0.1 & 1.9072 & 1.9059 & 1.9058 & $1.9172(2)$ \\
\hline
\end{tabular}

Table 2. $\frac{1}{N_{f}}\langle\bar{\psi} \psi\rangle$ with $N_{f}=4$ for various $m a$ including area 0 and 1 subdiagrams up to $L=4,6,8$.

The formulas needed to evaluate the chiral condensate at nonzero quark mass are given in (3.13)-(3.17). Taking into account the considerations in section 6.2, gives, considering up to $L=4$,

$$
\begin{aligned}
W_{a} & =\frac{2 d x}{1-\widehat{a}_{0} W_{a}-\widehat{b}_{0} W_{b}}, \\
W_{b} & =\frac{-4 d(d-1) x^{4} \frac{N_{f}}{N_{c}}}{\left(1-\widehat{a}_{1} W_{a}-\widehat{b}_{1}^{\prime} W_{b}\right)^{4}\left(1-\widehat{a}_{1} W_{a}-\widehat{b}_{1} W_{b}\right)^{2}\left(1-\widehat{a}_{1}^{\prime} W_{a}-\widehat{b}_{1} W_{b}\right)},
\end{aligned}
$$

where $x \equiv-\frac{1}{4 m^{2}}$. The results for $N_{f}=0$ are presented in table 1 , for $N_{f}=4$ in table 2 , along with the Monte Carlo data in [13]. The results considering diagrams using up to $L=6$, and $L=8$ area-1 sub-diagrams, are obtained analogously, with the appropriate mass-dependent equations for the $W_{x}$ in place of the $g_{x}$ in (7.14)-(7.16), and (7.17)-(7.20).

We note that when a non-zero quark mass is introduced, then the solutions for $\langle\bar{\psi} \psi\rangle$ which were each other's negative at zero quark mass separate and take different magnitudes, 
(a)

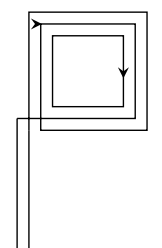

(d)

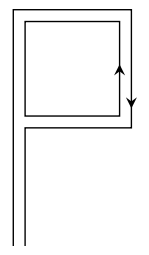

(b)

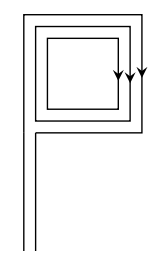

(c)

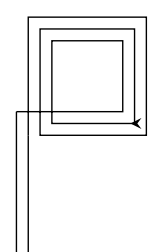

(e)

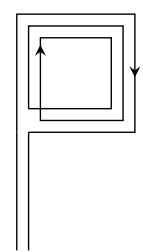

(f)

Figure 6. Lowest order diagrams used for constructing the set of reduced graphs.

therefore, there are four independent solutions (two positive, and two negative), instead of two. In the tables we present data for the solution which most closely matches the currently available lattice data for small $N_{f}$.

\subsection{Restricting to reduced graphs}

In order to compare with [12] we now examine the effects of allowing only reduced graphs, i.e. graphs where each closed loop is separated from all other closed loops as well as the origin by at least one double link. The set of reduced graphs can be obtained by modifying the diagrams used in the construction by inserting extra double links separating the loops. Perhaps the most remarkable difference in our approach from that of [12] is that in addition to the reduced graphs in [12], unreduced graphs are also included. We see no reason to discard these. Furthermore, due to the extra double links the reduced diagrams will have higher powers of $\left(a_{n} g_{a}+b_{n} g_{b}+\ldots\right)^{-1}$ and could as such be subdominant compared to the corresponding unreduced diagrams, by the arguments at the end of section 3. Regardless of this we will calculate the chiral condensate with a restriction to reduced graphs in order to compare with the results of [12].

The lowest order base diagrams in section 4 are modified as shown in figure 6 . These imply the following set of equations for the set of reduced graphs (where $g_{d}=0$ for $N_{c} \neq 3$ ).

$$
\begin{aligned}
g_{a} & =\frac{1}{a_{0} g_{a}+b_{0} g_{b}+b_{0} g_{d}}, \\
g_{b} & =-\frac{N_{f} / N_{c}}{\left(a_{1} g_{a}+d_{F} g_{b}+d_{F} g_{d}\right)^{8}\left(a_{1}^{\prime} g_{a}+d_{F} g_{b}+d_{F} g_{d}\right)}, \\
g_{d} & =-\frac{\frac{1}{3}\left(\frac{1}{2} N_{f}^{2}+2 N_{f}+1\right)}{\left(a_{1} g_{a}+d_{F} g_{b}+d_{F} g_{d}\right)^{12}\left(a_{1}^{\prime} g_{a}+d_{F} g_{b}+d_{F} g_{d}\right)},
\end{aligned}
$$

and

$$
\frac{\langle\bar{\psi} \psi\rangle}{N_{c} N_{f}}=\frac{2}{d_{a} g_{a}+d_{F} g_{b}+d_{F} g_{d}}
$$




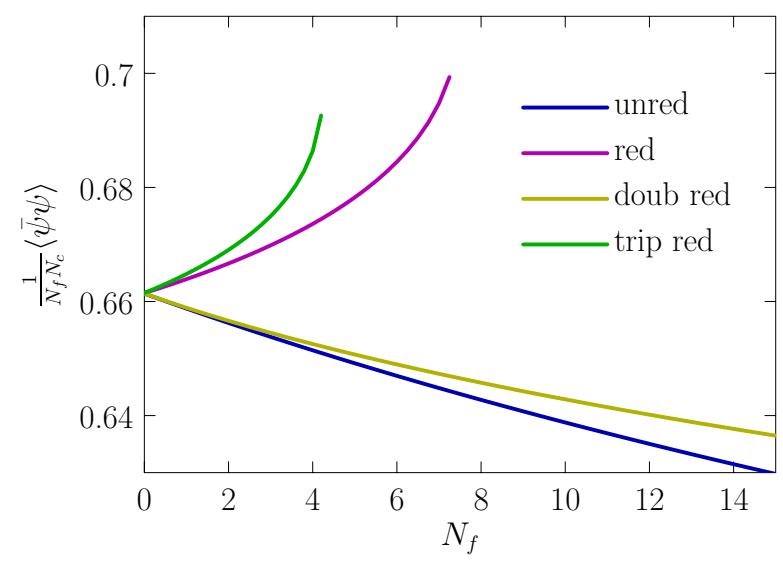

Figure 7. $\frac{1}{N_{f} N_{c}}\langle\bar{\psi} \psi\rangle$ for unreduced, reduced, doubly reduced, and triply reduced graphs.

where $d_{F}=8 d^{2}(d-1)$ is the dimensionality of a flag type diagram (figures 6b-6f). Solving these equations for $N_{c}=3$ and $d=4$ we find the chiral condensate for reduced graphs as shown in figure 7 compared to the condensate including the (partially) unreduced graphs built from the base diagrams of section $4 .^{7}$ We see that the $N_{f} \rightarrow 0$ limit is unchanged, as expected since the only diagrams that have been changed are those that depend on $N_{f}$. What is different is that when excluding unreduced graphs, the chiral condensate increases with $N_{f}$, and at $N_{f} \gtrsim 8$ staggered flavours it turns complex.

In order to examine closer the effects of excluding graphs in the recursive building we define a doubly (triply) reduced graph as a graph where each closed loop is separated from any other closed loop and the origin by at least two (three) tree segments and so on. Now if reduced graphs were in fact dominant, then by the same arguments, doubly reduced graphs (which are clearly also reduced) would be dominant among the reduced graphs. The set of doubly reduced graphs is generated by attaching an extra double link on the diagrams in figures $6 \mathrm{~b}-6 \mathrm{f}$, which leads to a change of the sign in equations (7.24) and (7.25) as well as an increase in the dimensionality $d_{F} \rightarrow 16 d^{3}(d-1)$. Restricting to doubly reduced graphs results in

$$
\begin{aligned}
g_{a} & =\frac{1}{a_{0} g_{a}+b_{0} g_{b}+b_{0} g_{d}}, \\
g_{b} & =\frac{N_{f} / N_{c}}{\left(a_{1} g_{a}+d_{F} g_{b}+d_{F} g_{d}\right)^{10}\left(a_{1}^{\prime} g_{a}+d_{F} g_{b}+d_{F} g_{d}\right)}, \\
g_{d} & =\frac{\frac{1}{3}\left(\frac{1}{2} N_{f}^{2}+2 N_{f}+1\right)}{\left(a_{1} g_{a}+d_{F} g_{b}+d_{F} g_{d}\right)^{14}\left(a_{1}^{\prime} g_{a}+d_{F} g_{b}+d_{F} g_{d}\right)} .
\end{aligned}
$$

The chiral condensate including only doubly reduced graphs (see figure 7) is again a decreasing function of $N_{f}$, which is real for all values of $N_{f}$, as was the case including unreduced diagrams, built from the base diagrams in section 4 .

\footnotetext{
${ }^{7}$ Note that figure 7 only shows one of the two solutions. For the second solution we do not observe a clear trend but the critical $N_{f}$ are the same in the cases where there is one.
} 
Going one step further and restricting to triply reduced graphs, the sign of $g_{b}$ and $g_{d}$ changes again,

$$
\begin{aligned}
g_{a} & =\frac{1}{a_{0} g_{a}+b_{0} g_{b}+b_{0} g_{d}}, \\
g_{b} & =-\frac{N_{f} / N_{c}}{\left(a_{1} g_{a}+d_{F} g_{b}+d_{F} g_{d}\right)^{12}\left(a_{1}^{\prime} g_{a}+d_{F} g_{b}+d_{F} g_{d}\right)}, \\
g_{d} & =-\frac{\frac{1}{3}\left(\frac{1}{2} N_{f}^{2}+2 N_{f}+1\right)}{\left(a_{1} g_{a}+d_{F} g_{b}+d_{F} g_{d}\right)^{16}\left(a_{1}^{\prime} g_{a}+d_{F} g_{b}+d_{F} g_{d}\right)},
\end{aligned}
$$

with $d_{F}=32 d^{4}(d-1)$. As shown in figure 7 , the chiral condensate becomes an increasing function of $N_{f}$ turning complex already for $N_{f} \geq 5$. This trend continues, such that for graphs reduced any even number of times we obtain a real decreasing condensate for all $N_{f}$, while for any odd number of double links separating the loops, the condensate turns complex at some critical $N_{f}$. This critical value of $N_{f}$ decreases with the number of required links, such that for quintuply reduced graphs and beyond the condensate is complex for all $N_{f} \gtrsim 1$.

This suggests that the existence of a critical $N_{f}$ above which the chiral condensate is complex as found in [12] is a direct consequence of the reduced approximation due to the change of sign in the equations for the $N_{f}$-dependent $g_{x}$ for type $x$-diagrams with an uneven number of double links attached to the loop diagrams. This is further supported by including (partially) unreduced graphs in the recursive building formulated instead as in [12]. This leads to a normalized chiral condensate that decreases with $N_{f}$ and remains real for all numbers of flavours.

\section{Discussion and conclusions}

In this paper, we have investigated an analytic lattice diagrammatic approach, that extends the work of $[8,9]$ to calculate the chiral condensate at infinite coupling, as a function of the number of flavours $N_{f}$. This work is motivated by recent simulation results [10], where a transition to chiral symmetry restoration is observed at a critical $N_{f} \sim 13$, contrary to the old analytic results of [7, 11]. It was suggested in [10] that the discrepancy between the simulations and the existing analytical results can be explained by noting that the latter incorrectly incorporate the $N_{f}$-dependence due to multiple meson hopping along a given link and baryon loops. A first analytical calculation to obtain the $N_{f}$-dependence of the chiral condensate at infinite coupling from a lattice diagrammatic approach was presented in [12]. There, a transition in the behavior of the chiral condensate was observed, in the sense that it turns complex at a critical value of $N_{f} \sim 10.7$ staggered flavours. While encouraging, the solution of [12], that connects to the reliable $N_{f} \rightarrow 0$ results of $[8,9]$ is not entirely satisfactory, as it increases with $N_{f}$, unlike what is observed in simulations. Motivated to understand this, we have explored a different approach than [12], in which we extend the lattice diagrammatic approach of $[8,9]$ by incorporating diagrams that capture the dominating $N_{f}$-dependence up to a given order in the hopping expansion. Let us stress that our approach is based on two approximations, namely the 
use of a hopping expansion and a restriction to area-1 (or less) sub-diagrams, which follows from the validity of the hopping expansion. The hopping expansion is well-motivated by $N_{f} \rightarrow 0$ results and is supported by simulation results. We have argued at the end of section 3 that sub-diagrams of area higher than 1 are generically of higher order and suppressed in comparison with the area-1 diagrams of the same order in $N_{f}$.

Overall, what we can conclude from our results for $\frac{1}{N_{f} N_{c}}\langle\bar{\psi} \psi\rangle$ is that the diagrammatic expansion appears to converge at smaller values of $N_{f}$, giving two real solutions in which the chiral condensate slowly approaches zero as a function of $N_{f}$, but does not exhibit discontinuities, other than the non-existence of solutions for non-integer $N_{f}$ in the $L=8$ truncation. The solution that approaches the results of $[8,9]$ as $N_{f} \rightarrow 0$ agrees quantitatively reasonably well with the simulation results of [13] for small values of $N_{f}$. The other solution is much larger at small $N_{f}$ and decreases more rapidly to zero at larger $N_{f}$.

We believe the differences from [12] are as follows. The mathematical formulation for the summing of diagrams is different, and we have included more contributions. Our calculations include higher order contributions up to $L=8$, [12] includes contributions up to $L=4$. Furthermore, additional diagrams are also included by allowing area 1 diagrams to attach directly to each other, resulting in "unreduced" and "partially reduced" graphs, using the terminology in [12]. Including these additional "unreduced" and "partially reduced" diagrams causes the transition to go away. In particular, if the base diagrams included in the resummation are in their most fundamental form (not decomposable into more fundamental base diagrams), then they all enter the formulation with a negative sign. ${ }^{8}$ While this appears to be the main reason for the lack of the transition in our results, and the decrease of the chiral condensate as a function of $N_{f}$, we note that counter-diagrams could potentially be included within our formulation, or that of [12], to correct for mis-counted overlapping diagrams, and some of these would come in with a positive sign.

Since we obtain quantitatively good results for the chiral condensate at small values of $N_{f}$, we view our results as encouraging and as a good first step in obtaining a reasonable $N_{f^{-}}$ dependence of the chiral condensate. We should however stress that it is at the moment difficult to see whether a transition to chiral symmetry restoration occurs at a critical value of $N_{f}$ in our approach, since all our solutions do not exhibit discontinuities. Such a transition is however not excluded a priori, as the chiral condensate might still jump between different solutions as $N_{f}$ is increased. Further work is therefore needed to elucidate the nature of a possible transition in the chiral condensate within our approach.

One possible avenue for further work and to improve on the $N_{f}$-dependence of the chiral condensate in our approach, consists of getting a better grip on various sources of error. Lattice diagrammatic approaches of the type used here and in [12], where non-tree contributions are included, are prone to sources of error associated with over-counting or miscounting of overlapping diagrams. We have dealt with various sources of error resulting from over-counting, however certain errors remain difficult to avoid. In particular, we note that the contribution of mistakes due to non-factorisation of integrations of overlapping

\footnotetext{
${ }^{8}$ In general, we have not been able to find a diagram (which is not a superposition) that has a different sign.
} 
diagrams could be important (see section 6.1) and we have not accounted for this effect in these results. In addition the effect of over-counting resulting from symmetries in winding diagrams (see section 6.3) should be investigated more thoroughly. This effect comes in at $L=6$ for $N_{c}=3$ and at $L=8$ for $N_{c}>3$. Finally, higher order graphs can become important at larger $N_{f}$ so there is still room for interesting behaviour in this regime. We leave the precise quantification of these errors for future research. It would be interesting to see whether such a precise quantification can shed more light on possible jumps of the chiral condensate between different solutions at a critical value of $N_{f}$. It would also be interesting for future work to see whether our approach can be extended to finite lattices. As the simulation results of [10] have been obtained on finite $4^{4}$ and $6^{4}$ lattices, this could offer an interesting possibility to allow for a better comparison of analytical approaches such as ours with simulation results.

Finally, note that higher dimensional representation fermions such as the symmetric, antisymmetric, and adjoint can also be considered in this approach. The calculation of diagrams with gauge fields in higher dimensional representations is however not simply a replacement of all instances of $N_{c}$ with $d_{R}$. This is an interesting topic which we are currently investigating.

\section{Acknowledgments}

We would like to thank Poul Damgaard, Matti Järvinen, Seyong Kim, Kim Splittorff, and Ben Svetitsky for useful discussions. JCM would like to thank the Sapere Aude program of the Danish Council for Independent Research for supporting this work. The work of JR is supported by the START project Y 435-N16 of the Austrian Science Fund (FWF).

\section{A Dimensionalities}

The dimensionalities $x_{n}$ are the number of ways to attach a diagram of type $x$ to a graph with area $n$ where the appropriate dimensionality is subtracted off $d_{x}$ to prevent overcounting, as explained in section 6.2. The dimensionalities in this section are relevant for the diagrams obtained in section 4 .

\begin{tabular}{|l|l|}
\hline$a_{0}$ & $2 d-1$ \\
\hline$b_{0}$ & $4(d-1)^{2}$ \\
\hline$d_{0}$ & $4(d-1)^{2}$ \\
\hline$g_{0}$ & $4(d-1)^{2}$ \\
\hline
\end{tabular}

\begin{tabular}{|l|l|}
\hline$a_{1}$ & $2 d-1$ \\
\hline$a_{1}^{\prime}$ & $2(d-1)$ \\
\hline$b_{1}$ & $4(d-1)^{2}$ \\
\hline$b_{1}^{\prime}$ & $4 d^{2}-6 d+1$ \\
\hline$d_{1}$ & $4(d-1)^{2}$ \\
\hline$d_{1}^{\prime}$ & $4 d^{2}-6 d+1$ \\
\hline$g_{1}$ & $4(d-1)^{2}$ \\
\hline$g_{1}^{\prime}$ & $4 d^{2}-6 d+1$ \\
\hline
\end{tabular}

The dimensional prefactors, $d_{x}$, correspond to the total number of ways an $x$-type diagram can be placed on the lattice. These are listed in the following table: 


\begin{tabular}{|l|l|}
\hline$d_{a}$ & $2 d$ \\
\hline$d_{b}$ & $4 d(d-1)$ \\
\hline$d_{c}$ & $12 d(d-1)(2 d-3)$ \\
\hline$d_{d}$ & $4 d(d-1)$ \\
\hline$d_{e}$ & $12 d(d-1)(2 d-3)$ \\
\hline$d_{f}$ & $48 d(d-1)(2 d-3)^{2}$ \\
\hline$d_{g}$ & $4 d(d-1)$ \\
\hline
\end{tabular}

\section{B Calculation of $I_{4}$}

The integral $I_{4}$

$$
I_{4}=\int_{\mathrm{SU}\left(N_{c}\right)} d U U_{a_{1}}^{b_{1}} U_{a_{2}}^{b_{2}} U_{a_{3}}^{b_{3}} U_{a_{4}}^{b_{4}}\left(U^{\dagger}\right)_{c_{1}}^{d_{1}}\left(U^{\dagger}\right)_{c_{2}}^{d_{2}}\left(U^{\dagger}\right)_{c_{3}}^{d_{3}}\left(U^{\dagger}\right)_{c_{4}}^{d_{4}}
$$

can be calculated as explained in section 5. One makes use of the decomposition

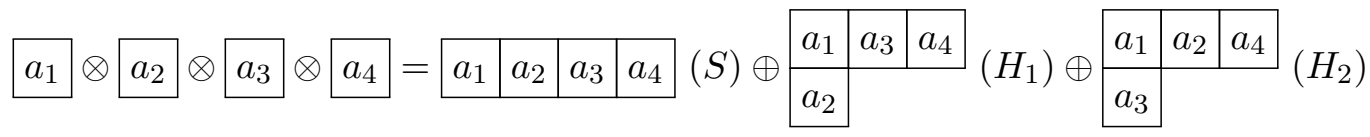

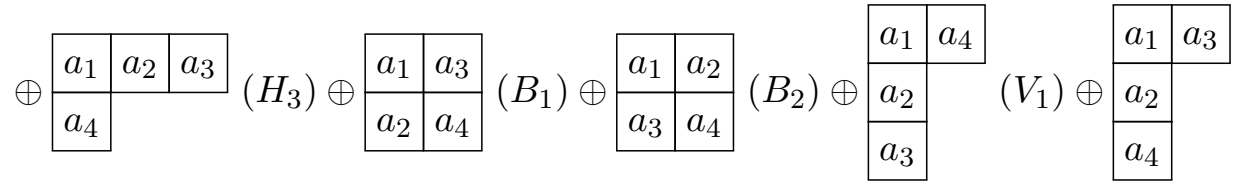

$$
\begin{aligned}
& \oplus \begin{array}{|l|l|}
\hline a_{1} & a_{2} \\
\hline a_{3} & \multirow{2}{|l|}{\quad\left(V_{3}\right) \oplus \begin{array}{|l|}
\hline a_{1} \\
\hline a_{2} \\
\hline a_{4}
\end{array}} \\
\hline a_{3} \\
\hline a_{4}
\end{array}(A) .
\end{aligned}
$$

In the following, the representations that appear in the right hand side of this equation will be denoted by the symbols in brackets (following their respective Young tableaux).

We can then define the Young projectors, that project onto the standard Young tableaux in the right hand side of the above equation. They are explicitly given by

$$
\begin{aligned}
& \mathbb{P}_{a_{1} a_{2} a_{3} a_{4}}^{S \quad b_{1} b_{2} b_{3} b_{4}}=\delta_{a_{1}}^{\left(b_{1}\right.} \delta_{a_{2}}^{b_{2}} \delta_{a_{3}}^{b_{3}} \delta_{a_{4}}^{\left.b_{4}\right)}, \\
& \mathbb{P}_{a_{1} a_{2} a_{3} a_{4}}^{H_{1} b_{1} b_{2} b_{3} b_{4}}=\frac{1}{8}\left(\delta_{a_{1}}^{b_{4}} \delta_{a_{2}}^{b_{2}} \delta_{a_{3}}^{b_{3}} \delta_{a_{4}}^{b_{1}}-\delta_{a_{1}}^{b_{2}} \delta_{a_{2}}^{b_{4}} \delta_{a_{3}}^{b_{3}} \delta_{a_{4}}^{b_{1}}+\delta_{a_{1}}^{b_{3}} \delta_{a_{2}}^{b_{2}} \delta_{a_{3}}^{b_{4}} \delta_{a_{4}}^{b_{1}}-\delta_{a_{1}}^{b_{2}} \delta_{a_{2}}^{b_{3}} \delta_{a_{3}}^{b_{4}} \delta_{a_{4}}^{b_{1}}\right. \\
& +\delta_{a_{1}}^{b_{4}} \delta_{a_{2}}^{b_{2}} \delta_{a_{3}}^{b_{1}} \delta_{a_{4}}^{b_{3}}-\delta_{a_{1}}^{b_{2}} \delta_{a_{2}}^{b_{4}} \delta_{a_{3}}^{b_{1}} \delta_{a_{4}}^{b_{3}}-\delta_{a_{1}}^{b_{2}} \delta_{a_{2}}^{b_{1}} \delta_{a_{3}}^{b_{4}} \delta_{a_{4}}^{b_{3}}+\delta_{a_{1}}^{b_{1}} \delta_{a_{2}}^{b_{2}} \delta_{a_{3}}^{b_{4}} \delta_{a_{4}}^{b_{3}} \\
& \left.+\delta_{a_{1}}^{b_{3}} \delta_{a_{2}}^{b_{2}} \delta_{a_{3}}^{b_{1}} \delta_{a_{4}}^{b_{4}}-\delta_{a_{1}}^{b_{2}} \delta_{a_{2}}^{b_{3}} \delta_{a_{3}}^{b_{1}} \delta_{a_{4}}^{b_{4}}-\delta_{a_{1}}^{b_{2}} \delta_{a_{2}}^{b_{1}} \delta_{a_{3}}^{b_{3}} \delta_{a_{4}}^{b_{4}}+\delta_{a_{1}}^{b_{1}} \delta_{a_{2}}^{b_{2}} \delta_{a_{3}}^{b_{3}} \delta_{a_{4}}^{b_{4}}\right), \\
& \mathbb{P}_{a_{1} a_{2} a_{3} a_{4}}^{H_{2} b_{1} b_{2} b_{3} b_{4}}=\frac{1}{8}\left(-\delta_{a_{1}}^{b_{3}} \delta_{a_{2}}^{b_{4}} \delta_{a_{3}}^{b_{2}} \delta_{a_{4}}^{b_{1}}+\delta_{a_{1}}^{b_{4}} \delta_{a_{2}}^{b_{2}} \delta_{a_{3}}^{b_{3}} \delta_{a_{4}}^{b_{1}}+\delta_{a_{1}}^{b_{2}} \delta_{a_{2}}^{b_{4}} \delta_{a_{3}}^{b_{3}} \delta_{a_{4}}^{b_{1}}-\delta_{a_{1}}^{b_{3}} \delta_{a_{2}}^{b_{2}} \delta_{a_{3}}^{b_{4}} \delta_{a_{4}}^{b_{1}}\right. \\
& -\delta_{a_{1}}^{b_{3}} \delta_{a_{2}}^{b_{4}} \delta_{a_{3}}^{b_{1}} \delta_{a_{4}}^{b_{2}}+\delta_{a_{1}}^{b_{4}} \delta_{a_{2}}^{b_{1}} \delta_{a_{3}}^{b_{3}} \delta_{a_{4}}^{b_{2}}+\delta_{a_{1}}^{b_{1}} \delta_{a_{2}}^{b_{4}} \delta_{a_{3}}^{b_{3}} \delta_{a_{4}}^{b_{2}}-\delta_{a_{1}}^{b_{3}} \delta_{a_{2}}^{b_{1}} \delta_{a_{3}}^{b_{4}} \delta_{a_{4}}^{b_{2}} \\
& \left.-\delta_{a_{1}}^{b_{3}} \delta_{a_{2}}^{b_{2}} \delta_{a_{3}}^{b_{1}} \delta_{a_{4}}^{b_{4}}-\delta_{a_{1}}^{b_{3}} \delta_{a_{2}}^{b_{1}} \delta_{a_{3}}^{b_{2}} \delta_{a_{4}}^{b_{4}}+\delta_{a_{1}}^{b_{2}} \delta_{a_{2}}^{b_{1}} \delta_{a_{3}}^{b_{3}} \delta_{a_{4}}^{b_{4}}+\delta_{a_{1}}^{b_{1}} \delta_{a_{2}}^{b_{2}} \delta_{a_{3}}^{b_{3}} \delta_{a_{4}}^{b_{4}}\right), \\
& \mathbb{P}_{a_{1} a_{2} a_{3} a_{4}}^{H_{3} b_{1} b_{2} b_{3} b_{4}}=\frac{1}{8}\left(-\delta_{a_{1}}^{b_{4}} \delta_{a_{2}}^{b_{3}} \delta_{a_{3}}^{b_{2}} \delta_{a_{4}}^{b_{1}}-\delta_{a_{1}}^{b_{4}} \delta_{a_{2}}^{b_{2}} \delta_{a_{3}}^{b_{3}} \delta_{a_{4}}^{b_{1}}-\delta_{a_{1}}^{b_{4}} \delta_{a_{2}}^{b_{3}} \delta_{a_{3}}^{b_{1}} \delta_{a_{4}}^{b_{2}}-\delta_{a_{1}}^{b_{4}} \delta_{a_{2}}^{b_{1}} \delta_{a_{3}}^{b_{3}} \delta_{a_{4}}^{b_{2}}\right.
\end{aligned}
$$




$$
\begin{aligned}
& -\delta_{a_{1}}^{b_{4}} \delta_{a_{2}}^{b_{2}} \delta_{a_{3}}^{b_{1}} \delta_{a_{4}}^{b_{3}}-\delta_{a_{1}}^{b_{4}} \delta_{a_{2}}^{b_{1}} \delta_{a_{3}}^{b_{2}} \delta_{a_{4}}^{b_{3}}+\delta_{a_{1}}^{b_{3}} \delta_{a_{2}}^{b_{2}} \delta_{a_{3}}^{b_{1}} \delta_{a_{4}}^{b_{4}}+\delta_{a_{1}}^{b_{2}} \delta_{a_{2}}^{b_{3}} \delta_{a_{3}}^{b_{1}} \delta_{a_{4}}^{b_{4}} \\
& \left.+\delta_{a_{1}}^{b_{3}} \delta_{a_{2}}^{b_{1}} \delta_{a_{3}}^{b_{2}} \delta_{a_{4}}^{b_{4}}+\delta_{a_{1}}^{b_{1}} \delta_{a_{2}}^{b_{3}} \delta_{a_{3}}^{b_{2}} \delta_{a_{4}}^{b_{4}}+\delta_{a_{1}}^{b_{2}} \delta_{a_{2}}^{b_{1}} \delta_{a_{3}}^{b_{3}} \delta_{a_{4}}^{b_{4}}+\delta_{a_{1}}^{b_{1}} \delta_{a_{2}}^{b_{2}} \delta_{a_{3}}^{b_{3}} \delta_{a_{4}}^{b_{4}}\right), \\
& \mathbb{P}_{a_{1} a_{2} a_{3} a_{4}}^{B_{1} b_{1} b_{2} b_{3} b_{4}}=\frac{1}{12}\left(\delta_{a_{1}}^{b_{4}} \delta_{a_{2}}^{b_{3}} \delta_{a_{3}}^{b_{2}} \delta_{a_{4}}^{b_{1}}-\delta_{a_{1}}^{b_{3}} \delta_{a_{2}}^{b_{4}} \delta_{a_{3}}^{b_{2}} \delta_{a_{4}}^{b_{1}}-\delta_{a_{1}}^{b_{3}} \delta_{a_{2}}^{b_{2}} \delta_{a_{3}}^{b_{4}} \delta_{a_{4}}^{b_{1}}+\delta_{a_{1}}^{b_{2}} \delta_{a_{2}}^{b_{3}} \delta_{a_{3}}^{b_{4}} \delta_{a_{4}}^{b_{1}}\right. \\
& -\delta_{a_{1}}^{b_{4}} \delta_{a_{2}}^{b_{3}} \delta_{a_{3}}^{b_{1}} \delta_{a_{4}}^{b_{2}}+\delta_{a_{1}}^{b_{3}} \delta_{a_{2}}^{b_{4}} \delta_{a_{3}}^{b_{1}} \delta_{a_{4}}^{b_{2}}-\delta_{a_{1}}^{b_{4}} \delta_{a_{2}}^{b_{1}} \delta_{a_{3}}^{b_{3}} \delta_{a_{4}}^{b_{2}}+\delta_{a_{1}}^{b_{1}} \delta_{a_{2}}^{b_{4}} \delta_{a_{3}}^{b_{3}} \delta_{a_{4}}^{b_{2}} \\
& +\delta_{a_{1}}^{b_{4}} \delta_{a_{2}}^{b_{1}} \delta_{a_{3}}^{b_{2}} \delta_{a_{4}}^{b_{3}}-\delta_{a_{1}}^{b_{1}} \delta_{a_{2}}^{b_{4}} \delta_{a_{3}}^{b_{2}} \delta_{a_{4}}^{b_{3}}+\delta_{a_{1}}^{b_{2}} \delta_{a_{2}}^{b_{1}} \delta_{a_{3}}^{b_{4}} \delta_{a_{4}}^{b_{3}}-\delta_{a_{1}}^{b_{1}} \delta_{a_{2}}^{b_{2}} \delta_{a_{3}}^{b_{4}} \delta_{a_{4}}^{b_{3}} \\
& \left.+\delta_{a_{1}}^{b_{3}} \delta_{a_{2}}^{b_{2}} \delta_{a_{3}}^{b_{1}} \delta_{a_{4}}^{b_{4}}-\delta_{a_{1}}^{b_{2}} \delta_{a_{2}}^{b_{3}} \delta_{a_{3}}^{b_{1}} \delta_{a_{4}}^{b_{4}}-\delta_{a_{1}}^{b_{2}} \delta_{a_{2}}^{b_{1}} \delta_{a_{3}}^{b_{3}} \delta_{a_{4}}^{b_{4}}+\delta_{a_{1}}^{b_{1}} \delta_{a_{2}}^{b_{2}} \delta_{a_{3}}^{b_{3}} \delta_{a_{4}}^{b_{4}}\right), \\
& \mathbb{P}_{a_{1} a_{2} a_{3} a_{4}}^{B_{2} b_{1} b_{2} b_{3} b_{4}}=\frac{1}{12}\left(\delta_{a_{1}}^{b_{4}} \delta_{a_{2}}^{b_{3}} \delta_{a_{3}}^{b_{2}} \delta_{a_{4}}^{b_{1}}+\delta_{a_{1}}^{b_{3}} \delta_{a_{2}}^{b_{4}} \delta_{a_{3}}^{b_{2}} \delta_{a_{4}}^{b_{1}}-\delta_{a_{1}}^{b_{2}} \delta_{a_{2}}^{b_{4}} \delta_{a_{3}}^{b_{3}} \delta_{a_{4}}^{b_{1}}-\delta_{a_{1}}^{b_{2}} \delta_{a_{2}}^{b_{3}} \delta_{a_{3}}^{b_{4}} \delta_{a_{4}}^{b_{1}}\right. \\
& +\delta_{a_{1}}^{b_{4}} \delta_{a_{2}}^{b_{3}} \delta_{a_{3}}^{b_{1}} \delta_{a_{4}}^{b_{2}}+\delta_{a_{1}}^{b_{3}} \delta_{a_{2}}^{b_{4}} \delta_{a_{3}}^{b_{1}} \delta_{a_{4}}^{b_{2}}-\delta_{a_{1}}^{b_{1}} \delta_{a_{2}}^{b_{4}} \delta_{a_{3}}^{b_{3}} \delta_{a_{4}}^{b_{2}}-\delta_{a_{1}}^{b_{1}} \delta_{a_{2}}^{b_{3}} \delta_{a_{3}}^{b_{4}} \delta_{a_{4}}^{b_{2}} \\
& -\delta_{a_{1}}^{b_{4}} \delta_{a_{2}}^{b_{2}} \delta_{a_{3}}^{b_{1}} \delta_{a_{4}}^{b_{3}}-\delta_{a_{1}}^{b_{4}} \delta_{a_{2}}^{b_{1}} \delta_{a_{3}}^{b_{2}} \delta_{a_{4}}^{b_{3}}+\delta_{a_{1}}^{b_{2}} \delta_{a_{2}}^{b_{1}} \delta_{a_{3}}^{b_{4}} \delta_{a_{4}}^{b_{3}}+\delta_{a_{1}}^{b_{1}} \delta_{a_{2}}^{b_{2}} \delta_{a_{3}}^{b_{4}} \delta_{a_{4}}^{b_{3}} \\
& \left.-\delta_{a_{1}}^{b_{3}} \delta_{a_{2}}^{b_{2}} \delta_{a_{3}}^{b_{1}} \delta_{a_{4}}^{b_{4}}-\delta_{a_{1}}^{b_{3}} \delta_{a_{2}}^{b_{1}} \delta_{a_{3}}^{b_{2}} \delta_{a_{4}}^{b_{4}}+\delta_{a_{1}}^{b_{2}} \delta_{a_{2}}^{b_{1}} \delta_{a_{3}}^{b_{3}} \delta_{a_{4}}^{b_{4}}+\delta_{a_{1}}^{b_{1}} \delta_{a_{2}}^{b_{2}} \delta_{a_{3}}^{b_{3}} \delta_{a_{4}}^{b_{4}}\right), \\
& \mathbb{P}_{a_{1} a_{2} a_{3} a_{4}}^{V_{1} b_{2} b_{3} b_{4}}=\frac{1}{8}\left(-\delta_{a_{1}}^{b_{4}} \delta_{a_{2}}^{b_{3}} \delta_{a_{3}}^{b_{2}} \delta_{a_{4}}^{b_{1}}+\delta_{a_{1}}^{b_{3}} \delta_{a_{2}}^{b_{4}} \delta_{a_{3}}^{b_{2}} \delta_{a_{4}}^{b_{1}}+\delta_{a_{1}}^{b_{4}} \delta_{a_{2}}^{b_{2}} \delta_{a_{3}}^{b_{3}} \delta_{a_{4}}^{b_{1}}-\delta_{a_{1}}^{b_{2}} \delta_{a_{2}}^{b_{4}} \delta_{a_{3}}^{b_{3}} \delta_{a_{4}}^{b_{1}}\right. \\
& -\delta_{a_{1}}^{b_{3}} \delta_{a_{2}}^{b_{2}} \delta_{a_{3}}^{b_{4}} \delta_{a_{4}}^{b_{1}}+\delta_{a_{1}}^{b_{2}} \delta_{a_{2}}^{b_{3}} \delta_{a_{3}}^{b_{4}} \delta_{a_{4}}^{b_{1}}-\delta_{a_{1}}^{b_{3}} \delta_{a_{2}}^{b_{2}} \delta_{a_{3}}^{b_{1}} \delta_{a_{4}}^{b_{4}}+\delta_{a_{1}}^{b_{2}} \delta_{a_{2}}^{b_{3}} \delta_{a_{3}}^{b_{1}} \delta_{a_{4}}^{b_{4}} \\
& \left.+\delta_{a_{1}}^{b_{3}} \delta_{a_{2}}^{b_{1}} \delta_{a_{3}}^{b_{2}} \delta_{a_{4}}^{b_{4}}-\delta_{a_{1}}^{b_{1}} \delta_{a_{2}}^{b_{3}} \delta_{a_{3}}^{b_{2}} \delta_{a_{4}}^{b_{4}}-\delta_{a_{1}}^{b_{2}} \delta_{a_{2}}^{b_{1}} \delta_{a_{3}}^{b_{3}} \delta_{a_{4}}^{b_{4}}+\delta_{a_{1}}^{b_{1}} \delta_{a_{2}}^{b_{2}} \delta_{a_{3}}^{b_{3}} \delta_{a_{4}}^{b_{4}}\right), \\
& \mathbb{P}_{a_{1} a_{2} a_{3} a_{4}}^{V_{2} b_{1} b_{2} b_{3} b_{4}}=\frac{1}{8}\left(-\delta_{a_{1}}^{b_{4}} \delta_{a_{2}}^{b_{2}} \delta_{a_{3}}^{b_{3}} \delta_{a_{4}}^{b_{1}}+\delta_{a_{1}}^{b_{2}} \delta_{a_{2}}^{b_{4}} \delta_{a_{3}}^{b_{3}} \delta_{a_{4}}^{b_{1}}+\delta_{a_{1}}^{b_{4}} \delta_{a_{2}}^{b_{3}} \delta_{a_{3}}^{b_{1}} \delta_{a_{4}}^{b_{2}}-\delta_{a_{1}}^{b_{3}} \delta_{a_{2}}^{b_{4}} \delta_{a_{3}}^{b_{1}} \delta_{a_{4}}^{b_{2}}\right. \\
& +\delta_{a_{1}}^{b_{4}} \delta_{a_{2}}^{b_{1}} \delta_{a_{3}}^{b_{3}} \delta_{a_{4}}^{b_{2}}-\delta_{a_{1}}^{b_{1}} \delta_{a_{2}}^{b_{4}} \delta_{a_{3}}^{b_{3}} \delta_{a_{4}}^{b_{2}}-\delta_{a_{1}}^{b_{4}} \delta_{a_{2}}^{b_{2}} \delta_{a_{3}}^{b_{1}} \delta_{a_{4}}^{b_{3}}+\delta_{a_{1}}^{b_{2}} \delta_{a_{2}}^{b_{4}} \delta_{a_{3}}^{b_{1}} \delta_{a_{4}}^{b_{3}} \\
& \left.+\delta_{a_{1}}^{b_{3}} \delta_{a_{2}}^{b_{2}} \delta_{a_{3}}^{b_{1}} \delta_{a_{4}}^{b_{4}}-\delta_{a_{1}}^{b_{2}} \delta_{a_{2}}^{b_{3}} \delta_{a_{3}}^{b_{1}} \delta_{a_{4}}^{b_{4}}-\delta_{a_{1}}^{b_{2}} \delta_{a_{2}}^{b_{1}} \delta_{a_{3}}^{b_{3}} \delta_{a_{4}}^{b_{4}}+\delta_{a_{1}}^{b_{1}} \delta_{a_{2}}^{b_{2}} \delta_{a_{3}}^{b_{3}} \delta_{a_{4}}^{b_{4}}\right), \\
& \mathbb{P}_{a_{1} a_{2} a_{3} a_{4}}^{V_{3} b_{1} b_{2} b_{3} b_{4}}=\frac{1}{8}\left(-\delta_{a_{1}}^{b_{4}} \delta_{a_{2}}^{b_{2}} \delta_{a_{3}}^{b_{3}} \delta_{a_{4}}^{b_{1}}+\delta_{a_{1}}^{b_{3}} \delta_{a_{2}}^{b_{2}} \delta_{a_{3}}^{b_{4}} \delta_{a_{4}}^{b_{1}}-\delta_{a_{1}}^{b_{4}} \delta_{a_{2}}^{b_{1}} \delta_{a_{3}}^{b_{3}} \delta_{a_{4}}^{b_{2}}+\delta_{a_{1}}^{b_{3}} \delta_{a_{2}}^{b_{1}} \delta_{a_{3}}^{b_{4}} \delta_{a_{4}}^{b_{2}}\right. \\
& +\delta_{a_{1}}^{b_{4}} \delta_{a_{2}}^{b_{2}} \delta_{a_{3}}^{b_{1}} \delta_{a_{4}}^{b_{3}}+\delta_{a_{1}}^{b_{4}} \delta_{a_{2}}^{b_{1}} \delta_{a_{3}}^{b_{2}} \delta_{a_{4}}^{b_{3}}-\delta_{a_{1}}^{b_{2}} \delta_{a_{2}}^{b_{1}} \delta_{a_{3}}^{b_{4}} \delta_{a_{4}}^{b_{3}}-\delta_{a_{1}}^{b_{1}} \delta_{a_{2}}^{b_{2}} \delta_{a_{3}}^{b_{4}} \delta_{a_{4}}^{b_{3}} \\
& \left.-\delta_{a_{1}}^{b_{3}} \delta_{a_{2}}^{b_{2}} \delta_{a_{3}}^{b_{1}} \delta_{a_{4}}^{b_{4}}-\delta_{a_{1}}^{b_{3}} \delta_{a_{2}}^{b_{1}} \delta_{a_{3}}^{b_{2}} \delta_{a_{4}}^{b_{4}}+\delta_{a_{1}}^{b_{2}} \delta_{a_{2}}^{b_{1}} \delta_{a_{3}}^{b_{3}} \delta_{a_{4}}^{b_{4}}+\delta_{a_{1}}^{b_{1}} \delta_{a_{2}}^{b_{2}} \delta_{a_{3}}^{b_{3}} \delta_{a_{4}}^{b_{4}}\right), \\
& \mathbb{P}_{a_{1} a_{2} a_{3} a_{4}}^{A b_{1} b_{2} b_{3} b_{4}}=\delta_{a_{1}}^{\left[b_{1}\right.} \delta_{a_{2}}^{b_{2}} \delta_{a_{3}}^{b_{3}} \delta_{a_{4}}^{\left.b_{4}\right]},
\end{aligned}
$$

where $(\cdots)$ and $[\cdots]$ in $\mathbb{P}^{S}$ and $\mathbb{P}^{A}$ denote complete symmetrization and antisymmetrization (with weight 1 , i.e. each term appears with a prefactor $1 / 24$ ) respectively.

Using these projectors, the matrix representatives of the irreducible representations $S$, $H_{1}, H_{2}, H_{3}, B_{1}, B_{2}, V_{1}, V_{2}, V_{3}, A$ can be constructed in terms of the matrices $U_{a}{ }^{b}$ in the fundamental representation

$$
\begin{aligned}
& S_{a_{1} a_{2} a_{3} a_{4}}^{b_{1} b_{2} b_{3} b_{4}}=\mathbb{P}_{a_{1} a_{2} a_{3} a_{4}}^{S \quad c_{1} c_{2} c_{3} c_{4}}\left(U_{c_{1}}^{d_{1}} U_{c_{2}}^{d_{2}} U_{c_{3}}^{d_{3}} U_{c_{4}}^{d_{4}}\right) \mathbb{P}_{d_{1} d_{2} d_{3} d_{4}}^{S b_{1} b_{2} b_{3} b_{4}}
\end{aligned}
$$

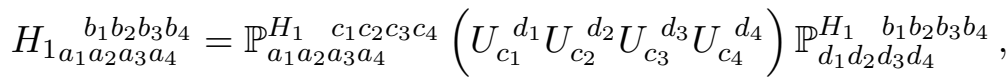

$$
\begin{aligned}
& H_{2}{ }_{a_{1} a_{2} a_{3} a_{4} b_{2} b_{3} b_{4}}^{b_{2}}=\mathbb{P}_{a_{1} a_{2} a_{3} a_{4} a_{4}}^{H_{2} c_{1} c_{2} c_{3} c_{4}}\left(U_{c_{1}}^{d_{1}} U_{c_{2}}^{d_{2}} U_{c_{3}}^{d_{3}} U_{c_{4}}^{d_{4}}\right) \mathbb{P}_{d_{1} d_{2} d_{3} d_{4}}^{H_{2} b_{1} b_{2} b_{3} b_{4}},
\end{aligned}
$$

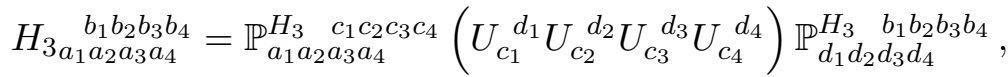

$$
\begin{aligned}
& B_{1 a_{1} a_{2} a_{3} a_{4}}^{b_{1} b_{2} b_{3} b_{4}}=\mathbb{P}_{a_{1} a_{2} a_{3} a_{1} a_{4}}^{B_{1} c_{1} c_{3} c_{4}}\left(U_{c_{1}}^{d_{1}} U_{c_{2}}^{d_{2}} U_{c_{3}}^{d_{3}} U_{c_{4}}^{d_{4}}\right) \mathbb{P}_{d_{1} d_{2} d_{3} d_{4}}^{B_{1} b_{1} b_{2} b_{3} b_{4}},
\end{aligned}
$$




$$
\begin{aligned}
& B_{2 a_{1} a_{2} a_{3} a_{4}}^{b_{1} b_{2} b_{3} b_{4}}=\mathbb{P}_{a_{1} a_{2} a_{3} a_{4}}^{c_{1} c_{2} c_{3} c_{4}}\left(U_{c_{1}}^{d_{1}} U_{c_{2}}^{d_{2}} U_{c_{3}}^{d_{3}} U_{c_{4}}^{d_{4}}\right) \mathbb{P}_{d_{1} d_{2} d_{3} d_{4}}^{B_{2} b_{1} b_{2} b_{3} b_{4}},
\end{aligned}
$$

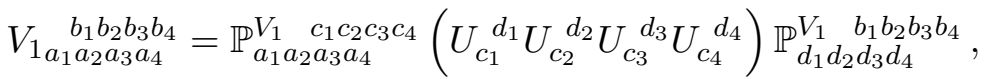

$$
\begin{aligned}
& V_{2 a_{1} a_{2} a_{3} a_{4}}^{b_{1} b_{2} b_{3} b_{4}}=\mathbb{P}_{a_{1} a_{2} a_{3} a_{4}}^{c_{2} c_{2} c_{3} c_{4}}\left(U_{c_{1}}^{d_{1}} U_{c_{2}}^{d_{2}} U_{c_{3}}^{d_{3}} U_{c_{4}}^{d_{4}}\right) \mathbb{P}_{d_{1} d_{2} d_{3} d_{4}}^{V_{2} b_{1} b_{2} b_{3} b_{4}} \\
& V_{3 a_{1} a_{2} a_{3} a_{4}}^{b_{1} b_{2} b_{3} b_{4}}=\mathbb{P}_{a_{1} a_{2} a_{3} a_{4}}^{V_{3} c_{2} c_{3} c_{4}}\left(U_{c_{1}}^{d_{1}} U_{c_{2}}^{d_{2}} U_{c_{3}}^{d_{3}} U_{c_{4}}^{d_{4}}\right) \mathbb{P}_{d_{1} d_{2} d_{3} d_{4}}^{V_{3} b_{1} b_{2} b_{3} b_{4}} \\
& A_{a_{1} a_{2} a_{3} a_{4}}^{b_{1} b_{2} b_{3} b_{4}}=\mathbb{P}_{a_{1} a_{2} a_{3} a_{4}}^{A c_{1} c_{2} c_{3} c_{4}}\left(U_{c_{1}}^{d_{1}} U_{c_{2}}^{d_{2}} U_{c_{3}}^{d_{3}} U_{c_{4}}^{d_{4}}\right) \mathbb{P}_{d_{1} d_{2} d_{3} d_{4}}^{A b_{1} b_{2} b_{3} b_{4}}
\end{aligned}
$$

One can then replace

$$
\begin{aligned}
& U_{a_{1}}^{b_{1}} U_{a_{2}}^{b_{2}} U_{a_{3}}^{b_{3}} U_{a_{4}}^{b_{4}}=S_{a_{1} a_{2} a_{3} a_{4}}^{b_{1} b_{2} b_{3} b_{4}}+H_{1 a_{1} a_{2} a_{3} a_{4}}^{b_{1} b_{2} b_{3} b_{4}}+H_{2 a_{1} a_{2} a_{3} a_{4}} b_{1} b_{2} b_{3} b_{4}+H_{3 a_{1} a_{2} a_{3} a_{4}} b_{1} b_{2} b_{3} b_{4}
\end{aligned}
$$

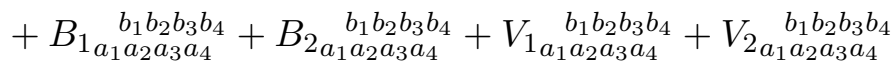

$$
\begin{aligned}
& +V_{3 a_{1} a_{2} a_{3} a_{4} b_{4} b_{3} b_{4}}+A_{a_{1} a_{2} a_{3} a_{4}}^{b_{1} b_{2} b_{3} b_{4}} \text {. }
\end{aligned}
$$

Using this in the original integral, one finds that it reduces to a sum of integrals of the form

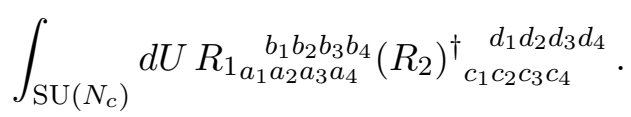

This integral is zero when $R_{1}$ and $R_{2}$ do not have the same Young tableau structure, so a lot of the terms vanish automatically. In particular, one gets the following non-zero contributions:

$$
\begin{aligned}
& \int_{\mathrm{SU}\left(N_{c}\right)} d U S_{a_{1} a_{2} a_{3} a_{4}}^{b_{1} b_{2} b_{3} b_{4}}(S)^{\dagger}{ }_{c_{1} c_{2} c_{3} c_{4}}^{d_{1} d_{2} d_{3} d_{4}}=\frac{24}{N_{c}\left(N_{c}+1\right)\left(N_{c}+2\right)\left(N_{c}+3\right)} \mathbb{P}_{a_{1} a_{2} a_{3} a_{4}}^{S \quad d_{1} d_{2} d_{3} d_{4}} \mathbb{P}_{c_{1} c_{2} c_{3} c_{4}}^{S b_{1} b_{2} b_{3} b_{4}}, \\
& \int_{\mathrm{SU}\left(N_{c}\right)} d U H_{1 a_{1} a_{2} a_{3} a_{4}}^{b_{1} b_{2} b_{3} b_{4}}\left(H_{1}\right)^{\dagger}{ }_{c_{1} c_{2} c_{3} c_{4}}^{d_{1} d_{2} d_{3} d_{4}}=\frac{8}{N_{c}\left(N_{c}^{2}-1\right)\left(N_{c}+2\right)} \mathbb{P}_{a_{1} a_{2} a_{3} a_{4}}^{H_{1} d_{1} d_{2} d_{3} d_{4}} \mathbb{P}_{c_{1} c_{2} c_{3} c_{4}}^{H_{1} b_{1} b_{2} b_{3} b_{4}}, \\
& \int_{\mathrm{SU}\left(N_{c}\right)} d U H_{2 a_{1} a_{2} a_{3} a_{4}}^{b_{1} b_{2} b_{3} b_{4}}\left(H_{2}\right)^{\dagger}{ }_{c_{1} c_{2} c_{3} c_{4}}^{d_{1} d_{2} d_{3} d_{4}}=\frac{8}{N_{c}\left(N_{c}^{2}-1\right)\left(N_{c}+2\right)} \mathbb{P}_{a_{1} a_{2} a_{3} a_{4}}^{H_{2} d_{1} d_{2} d_{3} d_{4}} \mathbb{P}_{c_{1} c_{2} c_{3} c_{4}}^{H_{2} b_{1} b_{2} b_{3} b_{4}}, \\
& \int_{\mathrm{SU}\left(N_{c}\right)} t d U H_{3 a_{1} a_{2} a_{3} a_{4}}^{b_{1} b_{2} b_{3} b_{4}}\left(H_{3}\right)^{\dagger}{ }_{c_{1} c_{2} c_{3} c_{4}}^{d_{1} d_{2} d_{3} d_{4}}=\frac{8}{N_{c}\left(N_{c}^{2}-1\right)\left(N_{c}+2\right)} \mathbb{P}_{a_{1} a_{2} a_{3} a_{4}}^{H_{3} d_{1} d_{2} d_{3} d_{4}} \mathbb{P}_{c_{1} c_{2} c_{3} c_{4}}^{H_{3} b_{1} b_{2} b_{3} b_{4}}, \\
& \int_{\mathrm{SU}\left(N_{c}\right)} d U H_{1 a_{1} a_{2} a_{3} a_{4}}^{b_{1} b_{2} b_{3} b_{4}}\left(H_{2}\right)^{\dagger}{ }_{c_{1} c_{2} c_{3} c_{4}}^{d_{1} d_{2} d_{3} d_{4}}=\frac{8}{N_{c}\left(N_{c}^{2}-1\right)\left(N_{c}+2\right)} \mathbb{P}_{a_{1} a_{2} a_{3} a_{4}}^{H_{1} d_{1} d_{3} d_{2} d_{4}} \mathbb{P}_{c_{1} c_{2} c_{3} c_{4}}^{H_{2} b_{3} b_{3} b_{2} b_{4}}, \\
& \int_{\mathrm{SU}\left(N_{c}\right)} d U H_{1 a_{1} a_{2} a_{3} a_{4}}^{b_{1} b_{2} b_{3} b_{4}}\left(H_{3}\right)^{\dagger}{ }_{c_{1} c_{2} c_{3} c_{4}}^{d_{1} d_{2} d_{3} d_{4}}=\frac{8}{N_{c}\left(N_{c}^{2}-1\right)\left(N_{c}+2\right)} \mathbb{P}_{a_{1} a_{2} a_{3} a_{4}}^{H_{1} d_{1} d_{4} d_{2} d_{3}} \mathbb{P}_{c_{1} c_{2} c_{3} c_{4}}^{H_{3} b_{3} b_{3} b_{4} b_{2}}, \\
& \int_{\mathrm{SU}\left(N_{c}\right)} d U H_{2}^{a_{a_{1} a_{2} a_{3} a_{2} b_{2} b_{3} b_{4}}}\left(H_{1}\right)^{\dagger}{ }_{c_{1} c_{2} c_{3} c_{4}}^{d_{1} d_{2} d_{3} d_{4}}=\frac{8}{N_{c}\left(N_{c}^{2}-1\right)\left(N_{c}+2\right)} \mathbb{P}_{a_{1} a_{2} a_{3} a_{4}}^{H_{2} d_{1} d_{3} d_{2} d_{4}} \mathbb{P}_{c_{1} c_{2} c_{3} c_{4}}^{H_{1} b_{1} b_{3} b_{2} b_{4}},
\end{aligned}
$$

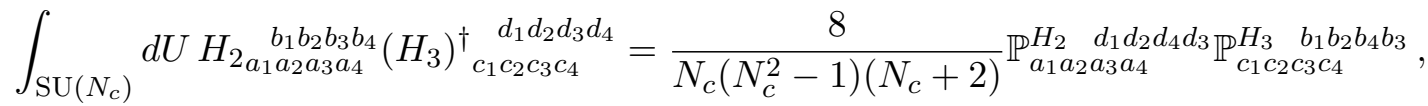

$$
\begin{aligned}
& \int_{\mathrm{SU}\left(N_{c}\right)} d U H_{3 a_{1} a_{2} a_{3} a_{4}}^{b_{1} b_{2} b_{3} b_{4}}\left(H_{1}\right)^{\dagger}{ }_{c_{1} c_{2} c_{3} c_{4}}^{d_{1} d_{2} d_{3} d_{4}}=\frac{8}{N_{c}\left(N_{c}^{2}-1\right)\left(N_{c}+2\right)} \mathbb{P}_{a_{1} a_{2} a_{3} a_{4}}^{H_{3} \quad d_{1} d_{3} d_{4} d_{2}} \mathbb{P}_{c_{1} c_{2} c_{3} c_{4}}^{H_{1} \quad b_{1} b_{4} b_{2} b_{3}}, \\
& \int_{\mathrm{SU}\left(N_{c}\right)} d U H_{3 a_{1} a_{2} a_{3} a_{3} b_{2} b_{3} b_{4}}\left(H_{2}\right)^{\dagger}{ }_{c_{1} c_{2} c_{3} c_{4}}^{d_{1} d_{2} d_{3} d_{4}}=\frac{8}{N_{c}\left(N_{c}^{2}-1\right)\left(N_{c}+2\right)} \mathbb{P}_{a_{1} a_{2} a_{3} a_{4}}^{H_{3} d_{1} d_{2} d_{4} d_{3}} \mathbb{P}_{c_{1} c_{2} c_{3} c_{4}}^{H_{2} b_{1} b_{2} b_{4} b_{3}},
\end{aligned}
$$

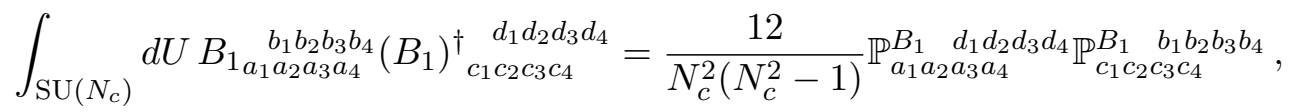




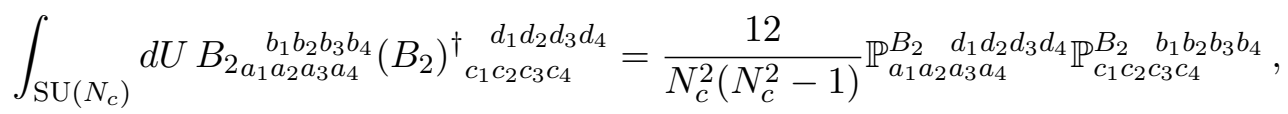

$$
\begin{aligned}
& \int_{\mathrm{SU}\left(N_{c}\right)} d U B_{1 a_{1} a_{2} a_{3} a_{4}}^{b_{1} b_{2} b_{3} b_{4}}\left(B_{2}\right)^{\dagger}{ }_{c_{1} c_{2} c_{3} c_{4}}^{d_{1} d_{2} d_{3} d_{4}}=\frac{12}{N_{c}^{2}\left(N_{c}^{2}-1\right)} \mathbb{P}_{a_{1} a_{2} a_{3} a_{4}}^{B_{1} d_{1} d_{3} d_{2} d_{4}} \mathbb{P}_{c_{1} c_{2} c_{3} c_{4}}^{B_{2}} b_{1} b_{3} b_{2} b_{4},
\end{aligned}
$$

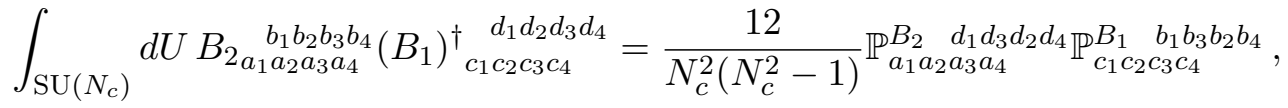

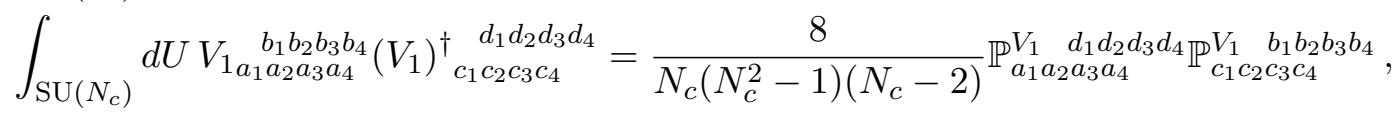

$$
\begin{aligned}
& \int_{\mathrm{SU}\left(N_{c}\right)} d U V_{2 a_{1} a_{2} a_{3} a_{4}}^{b_{1} b_{2} b_{3} b_{4}}\left(V_{2}\right)^{\dagger}{ }_{c_{1} c_{2} c_{3} c_{4}}^{d_{1} d_{2} d_{3} d_{4}}=\frac{8}{N_{c}\left(N_{c}^{2}-1\right)\left(N_{c}-2\right)} \mathbb{P}_{a_{1} a_{2} a_{3} a_{4}}^{d_{2} d_{1} d_{2} d_{3} d_{4}} \mathbb{P}_{c_{1} c_{2} c_{3} c_{4}}^{V_{2} b_{1} b_{2} b_{3} b_{4}}, \\
& \int_{\mathrm{SU}\left(N_{c}\right)} d U V_{3 a_{1} a_{2} a_{3} a_{4}}^{b_{1} b_{2} b_{3} b_{4}}\left(V_{3}\right)^{\dagger}{ }_{c_{1} c_{2} c_{3} c_{4}}^{d_{1} d_{2} d_{3} d_{4}}=\frac{8}{N_{c}\left(N_{c}^{2}-1\right)\left(N_{c}-2\right)} \mathbb{P}_{a_{1} a_{2} a_{3} a_{4}}^{V_{3} d_{1} d_{2} d_{3} d_{4}} \mathbb{P}_{c_{1} c_{2} c_{3} c_{4}}^{V_{3} b_{1} b_{2} b_{3} b_{4}},
\end{aligned}
$$

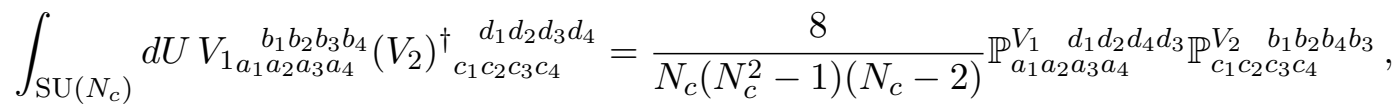

$$
\begin{aligned}
& \int_{\mathrm{SU}\left(N_{c}\right)} d U V_{1 a_{1} a_{2} a_{3} a_{4}}^{b_{1} b_{2} b_{3} b_{4}}\left(V_{3}\right)^{\dagger}{ }_{c_{1} c_{2} c_{3} c_{4}}^{d_{1} d_{2} d_{3} d_{4}}=\frac{8}{N_{c}\left(N_{c}^{2}-1\right)\left(N_{c}-2\right)} \mathbb{P}_{a_{1} a_{2} a_{3} a_{4}}^{V_{1} d_{1} d_{3} d_{4} d_{2}} \mathbb{P}_{c_{1} c_{2} c_{3} c_{4}}^{V_{3} b_{1} b_{4} b_{2} b_{3}}, \\
& \int_{\mathrm{SU}\left(N_{c}\right)} d U V_{2 a_{1} a_{2} a_{3} a_{4}}^{b_{1} b_{2} b_{3} b_{4}}\left(V_{1}\right)^{\dagger}{ }_{c_{1} c_{2} c_{3} c_{4}}^{d_{1} d_{2} d_{3} d_{4}}=\frac{8}{N_{c}\left(N_{c}^{2}-1\right)\left(N_{c}-2\right)} \mathbb{P}_{a_{1} a_{2} a_{3} a_{4}}^{d_{2} d_{1} d_{2} d_{4} d_{3}} \mathbb{P}_{c_{1} c_{2} c_{3} c_{4}}^{V_{1} b_{1} b_{2} b_{4} b_{3}}, \\
& \int_{\mathrm{SU}\left(N_{c}\right)} d U V_{2}{ }_{a_{1} a_{2} a_{3} a_{4}}^{b_{1} b_{2} b_{3} b_{4}}\left(V_{3}\right)^{\dagger}{ }_{c_{1} c_{2} c_{3} c_{4}}^{d_{1} d_{2} d_{3} d_{4}}=\frac{8}{N_{c}\left(N_{c}^{2}-1\right)\left(N_{c}-2\right)} \mathbb{P}_{a_{1} a_{2} a_{3} a_{4}}^{V_{2} d_{1} d_{3} d_{2} d_{4}} \mathbb{P}_{c_{1} c_{2} c_{3} c_{4}}^{V_{3} b_{1} b_{3} b_{2} b_{4}}, \\
& \int_{\mathrm{SU}\left(N_{c}\right)} d U V_{3 a_{1} a_{2} a_{3} a_{4}}^{b_{1} b_{2} b_{3} b_{4}}\left(V_{1}\right)^{\dagger}{ }_{c_{1} c_{2} c_{3} c_{4}}^{d_{1} d_{2} d_{3} d_{4}}=\frac{8}{N_{c}\left(N_{c}^{2}-1\right)\left(N_{c}-2\right)} \mathbb{P}_{a_{1} a_{2} a_{3} a_{4}}^{V_{3} d_{1} d_{4} d_{2} d_{3}} \mathbb{P}_{c_{1} c_{2} c_{3} c_{4}}^{V_{1} b_{1} b_{3} b_{4} b_{2}}, \\
& \int_{\mathrm{SU}\left(N_{c}\right)} d U V_{3 a_{1} a_{2} a_{3} a_{4}}^{b_{1} b_{2} b_{3} b_{4}}\left(V_{2}\right)^{\dagger}{ }_{c_{1} c_{2} c_{3} c_{4}}^{d_{1} d_{2} d_{3} d_{4}}=\frac{8}{N_{c}\left(N_{c}^{2}-1\right)\left(N_{c}-2\right)} \mathbb{P}_{a_{1} a_{2} a_{3} a_{4} a_{1} d_{3} d_{2} d_{4}}^{V_{4} \mathbb{P}_{2} b_{1} b_{1} b_{3} b_{2} b_{4}}, \\
& \int_{\mathrm{SU}\left(N_{c}\right)} d U A_{a_{1} a_{2} a_{3} a_{4}}^{b_{1} b_{2} b_{3} b_{4}} A_{c_{1} c_{2} c_{3} c_{4}}^{d_{1} d_{2} d_{3} d_{4}}=\frac{24}{N_{c}\left(N_{c}-1\right)\left(N_{c}-2\right)\left(N_{c}-3\right)} \mathbb{P}_{a_{1} a_{2} a_{3} a_{4}}^{A d_{1} d_{2} d_{3} d_{4}} \mathbb{P}_{c_{1} c_{2} c_{3} c_{4}}^{A} b_{1} b_{2} b_{3} b_{4}
\end{aligned}
$$

The final result for $I_{4}$ is then given by the sum of all the above terms.

Open Access. This article is distributed under the terms of the Creative Commons Attribution License (CC-BY 4.0), which permits any use, distribution and reproduction in any medium, provided the original author(s) and source are credited.

\section{References}

[1] A. Deuzeman, M.P. Lombardo, T. Nunes Da Silva and E. Pallante, The bulk transition of QCD with twelve flavors and the role of improvement, Phys. Lett. B 720 (2013) 358 [arXiv:1209.5720] [INSPIRE].

[2] A. Cheng, A. Hasenfratz, Y. Liu, G. Petropoulos and D. Schaich, Finite size scaling of conformal theories in the presence of a near-marginal operator, Phys. Rev. D 90 (2014) 014509 [arXiv: 1401.0195] [INSPIRE].

[3] Z. Fodor et al., Twelve massless flavors and three colors below the conformal window, Phys. Lett. B 703 (2011) 348 [arXiv:1104.3124] [INSPIRE]. 
[4] C.-J.D. Lin, K. Ogawa, H. Ohki and E. Shintani, Lattice study of infrared behaviour in SU(3) gauge theory with twelve massless flavours, JHEP 08 (2012) 096 [arXiv:1205.6076] [INSPIRE].

[5] E. Itou, The twisted Polyakov loop coupling and the search for an IR fixed point, PoS (LATTICE 2013) 005 [arXiv: 1311.2676] [INSPIRE].

[6] F. Bursa, L. Del Debbio, L. Keegan, C. Pica and T. Pickup, Mass anomalous dimension in SU(2) with six fundamental fermions, Phys. Lett. B 696 (2011) 374 [arXiv:1007.3067] [INSPIRE].

[7] H. Kluberg-Stern, A. Morel and B. Petersson, Spectrum of lattice gauge theories with fermions from a $1 / D$ expansion at strong coupling, Nucl. Phys. B 215 (1983) 527 [INSPIRE].

[8] J.M. Blairon, R. Brout, F. Englert and J. Greensite, Chiral symmetry breaking in the action formulation of lattice gauge theory, Nucl. Phys. B 180 (1981) 439 [INSPIRE].

[9] O. Martin and B. Siu, Chiral symmetry breaking in strongly coupled lattice gauge theory, Phys. Lett. B 131 (1983) 419 [INSPIRE].

[10] P. de Forcrand, S. Kim and W. Unger, Conformality in many-flavour lattice QCD at strong coupling, JHEP 02 (2013) 051 [arXiv: 1208.2148] [INSPIRE].

[11] P.H. Damgaard, D. Hochberg and N. Kawamoto, Effective Lagrangian analysis of the chiral phase transition at finite density, Phys. Lett. B 158 (1985) 239 [INSPIRE].

[12] E.T. Tomboulis, Absence of chiral symmetry breaking in multi-flavor strongly coupled lattice gauge theories, Phys. Rev. D 87 (2013) 034513 [arXiv:1211.4842] [INSPIRE].

[13] P. de Forcrand and S. Kim, The spectrum of lattice QCD with staggered fermions at strong coupling, Phys. Lett. B 645 (2007) 339 [hep-lat/0608012] [INSPIRE].

[14] I. Bars and F. Green, Complete integration of $\mathrm{U}(N)$ lattice gauge theory in a large- $N$ limit, Phys. Rev. D 20 (1979) 3311 [INSPIRE].

[15] M. Creutz, Quarks, gluons and lattices, Cambridge Monographs on Mathematical Physics, Cambridge University Press, Cambridge U.K. (1985).

[16] P. Cvitanovic, Group theory: birdtracks, Lie's and exceptional groups, Princeton University Press, Princeton U.S.A. (2008).

[17] K.G. Wilson, Quarks and strings on a lattice, CLNS-321, (1975) [INSPIRE].

[18] J.G. Simmonds and J.E. Mann, A first look at perturbation theory, Dover Publications, U.S.A. (1986).

[19] C.M. Bender and S.A. Orszag, Advanced mathematical methods for scientists and engineers, McGraw-Hill, U.S.A. (1978). 